\title{
Comment on Clebsch's 1857 and 1859 papers on using Hamiltonian methods in hydrodynamics
}

\author{
Gérard Grimberg \\ Instituto de Matematica - UFRJ, Av. Athos da Silveira Ramos 149, CT - Bloco C, \\ Cid. Univ. - Ilha do Fundão. Caixa Postal 68530 21941-909 Rio de Janeiro - RJ - Brasil \\ Emanuele Tassi * \\ Université Côte d'Azur, Observatoire de la Côte d'Azur, CNRS, Laboratoire J. L. Lagrange, \\ Boulevard de l'Observatoire, CS 34229, 06304 Nice Cedex 4, France
}

\begin{abstract}
The present paper is a companion of two translated articles by Alfred Clebsch, titled "On a general transformation of the hydrodynamical equations" and "On the integration of the hydrodynamical equations". The originals were published in the Journal für die reine and angewandte Mathematik" (1857 and 1859). Here we provide a detailed critical reading of these articles, which analyzes methods, and results of Clebsch. In the first place, we try to elucidate the algebraic calculus used by Clebsch in several parts of the two articles that we believe to be the most significant ones. We also provide some proofs that Clebsch did not find necessary to explain, in particular concerning the variational principles stated in his two articles and the use of the method of Jacobi's Last Multiplier. When possible, we reformulate the original expressions by Clebsch in the language of vector analysis, which should be more familiar to the reader. The connections of the results and methods by Clebsch with his scientific context, in particular with the works of Carl Jacobi, are briefly discussed. We emphasize how the representations of the velocity vector field conceived by Clebsch in his two articles, allow for a variational formulation of hydrodynamics equations in the steady and unsteady case. In particular, we stress that what is nowadays known as the "Clebsch variables", permit to give a canonical Hamiltonian formulation of the equations of fluid mechanics. We also list a number of further developments of the theory initiated by Clebsch, which had an impact on presently active areas of research, within such fields as hydrodynamics and plasma physics.
\end{abstract}

\section{INTRODUCTION}

The two articles, which are commented here, present early results by Alfred Clebsch with relatively little immediate impact on the development of hydrodynamics. Besides the little known work - at that time - by Hankel, some results by Beltrami and remarks in articles by Basset, Hicks, Lamb and Duhem, which were based on the two articles by Clebsch, the results of the German mathematician on the variational formulation of hydrodynamics equations, did not lead to any major development, in the decades immediately following the years of their publication. ${ }^{1}$

Since the years 1929-30, a few related articles appear, namely those by Bateman, and by Herivel. A renewed interest in the works by Clebsch occurs then in the 1960s, not only in connection with hydrodynamics, as done by Lin, and also by Bretherton, but also with regard to magnetohydrodynamics and electromagnetism, as in the work by Calkin, and in the article by Seliger and Whitham. ${ }^{2}$

Finally, starting from the 1980s, we observe a much more important number of results related to the two articles by Clebsch and, in particular, to the representation of vector fields in terms of the variables that Clebsch introduced in his 1859 paper. It is practically impossible to provide an exhaustive list of the results obtained during the last four decades and stemming from the introduction of Clebsch variables. In Sec. V, we attempt to provide, however, a number of references exemplifying various recent applications of Clebsch variables, and of their extensions, to theoretical and mathematical physics, in particular, concerning hydrodynamics and plasma physics.

In the two papers under consideration, regarding historical reasons for the motivations and the methods adopted (in particular under the influence of Carl Jacobi's work), it is

\footnotetext{
* e-mail : etassi@oca.eu

1 Hankel 1861, Beltrami 1871, Basset 1888, Hicks 1882, Lamb 1895, Duhem 1901.

2 Bateman 1929, Herivel 1954, 1955, Calkin 1963, Lin 1963, Bretherton 1970, Seliger 1968.
} 
worth summarizing here a few biographical notes about Clebsch's childhood and youth.

During his childhood, Clebsch became friend with Karl Neumann, the son of Franz Neumann. In her biography on Peter Gustav Lejeune Dirichlet, Uta Merzbach, who reports about the visit of Dirichlet to Carl Jacobi in 1843, mentions the presence of Clebsch (who was 10 years old at that time) and of his friend Karl at family meetings. At the university, Clebsch took the Physics course taught by Franz Neumann (friend and collaborator of Jacobi), as well as other courses taught by Jacobi's disciples, namely Friedrich Julius Richelot (Advanced Geometry, Partial Differential Equations, Mechanics) and Otto Hesse (Mechanics, Integral Calculus and Variational Calculus ). ${ }^{3}$

When Clebsch completed his doctoral thesis at the University of Königsberg in 1954, he was just 21 years old. His supervisor is the previously mentioned physicist Franz Neumann. Clebsch's doctoral thesis concerns the motion of an ellipsoid in an incompressible fluid with a potential velocity field. Clebsch treated two separate cases, corresponding to the situations when the body executes a translational or a rotational motion, respectively. This work is published, with some modifications, in Crelle's journal, in German, and is followed by a note published one year later. The authors that Clebsch cites (Siméon-Denis Poisson, Dirichlet, Gabriel Lamé, Jacobi) and the methods that he adopts, show that he is aware of the most recent works on the subject and that hydroynamics is at center of his scientific interests during the years immediately following his thesis. ${ }^{4}$

In 1855 Clebsch moves to Berlin, where he writes his Habilitation thesis, which he defends in 1858. During this period Clebsch publishes several articles in Crelle's Journal that, since 1855, is edited by Borchardt, former student of Dirichlet and Jacobi. After Jacobi's death in 1851, Dirichlet, who inherits all Jacobi's manuscripts, takes care of their publication, in collaboration with Carl Borchardt. Some of these articles, including from Clebsch, are published in Crelle's Journal. In 1862 he edits, in Crelle's Journal, the famous manuscript of Jacobi about Pfaff's problem (where the Jacobi identity appears, in relation with the Poisson bracket) and, a little later, in 1866, according to Borchardt's notes, the Vorlesungen über Dynamik, which consist of courses given by Jacobi at the university of Königsberg in winter 1842 -1843. In the following year, Jacobi, ill, leaves Königsberg and will never come back. However, we can suppose that the content of his Vorlesungen made use of courses taught by Jacobi and written down by Clebsch and also taught by Richelot and Hesse. Also, thanks to the Crelle's journal and the publication, in two volumes, of the most important mathematical articles by Jacobi, Clebsch becomes familiar with the main results by Jacobi. ${ }^{5}$

We finally remark that, during the period from 1856 to 1859, Clebsch published in Crelle's Journal seven articles and one in Monatsberichte der Königlichen Preussische Akademie des Wissenschaften zu Berlin, related to the subject of interest for the present paper. Four of them concerned hydrodynamics and four deal with calculus of variations. Also connected with the two articles that we are considering, are the articles on Pfaff's problem belonging to the period from 1861 to $1863 .{ }^{6}$

Our paper is organized as follows. Section II describes the context of Clebsch 1857 and 1859 papers, and their key results.

Sections III and IV describe the results and methods of the article of 1857 and 1859, respectively. Besides elucidating and commenting a number of algebraic steps of Clebsch's papers, we explain and emphasize in particular, the use of the method of Last Multiplier and recall the canonical Hamiltonian formulation of Euler's equation for an incompressible fluid, which emerges from the introduction of Clebsch variables.

Concluding remarks are presented in Section V, with some observations, in particular, about more recent developments and applications of the vector field decomposition that Clebsch introduced in his 1859 paper.

\footnotetext{
3 Merzbach 2018:136, Clebsch 1854:17-18.

4 Clebsch 1854, 1856, 1857a.

5 Jacobi 1846, 1851.

6 Clebsch 1856, 1857a, 1857b, 1858a, 1858b, 1858c, 1859a, 1859b, 1861, 1862, 1863. For detailed biographical information on Clebsch, see Burau, 1970-1980 and Various Authors, 1873a; 1873b.
} 


\section{CONTEXT OF CLEBSCH'S 1857 AND 1859 ARTICLES ON HYDRODYNAMICS AND MOST IMPORTANT RESULTS}

It is particularly interesting to consider in a somewhat detailed manner the results by Jacobi that Clebsch applies to hydrodynamics. One of the fundamental articles in this context is Theoria Novi Multiplicatoris ad varia exempla applicata, published by Jacobi in 1844, which describes his method of the Last Multiplier. Jacobi and two of his disciples, Kummer and Clebsch, had a way to denote what we now call $n$-dimensional (vector) spaces, using, one scalar field and $n-1$ vector fields. There some cases where the notation has an interesting geometric interpretation. ${ }^{7}$

Anyway, we find it more convenient to switch to modern notation. We shall return to this matter when presenting the Clebsch notation for multi-dimensionl problems (see Sec. III.A). Jacobi's method is based on a lemma and on a theorem. The "Fundamental Lemma" by Jacobi can be stated in the following way: Let $f_{1}, f_{2}, \cdots, f_{n}$ be $n$ functions of $n$ variables $x_{1}, x_{2}, \cdots, x_{n}$. The co-factors $A_{i j}$, with $i, j=1, \ldots, n$, of the terms $\partial_{x_{j}} f_{i}$ of the Jacobian matrix $\frac{\partial\left(f_{1}, f_{2}, \cdots, f_{n}\right)}{\partial\left(x_{1}, \cdots, x_{n}\right)}$ satisfy $\sum_{j=1}^{n} \partial_{x_{j}} A_{i j}=0$ for $i=1, \cdots, n$.

This lemma allows Jacobi to prove the following theorem: let $\mathbf{X}=\left(X_{1}, \cdots, X_{n}\right)$ be a vector field where $X_{1}, \cdots, X_{n}$ are $n$ functions of $x_{1}, \cdots, x_{n}$. The $(n-1)$ th integral of the system $\frac{d x_{1}}{X_{1}}=\cdots=\frac{d x_{n}}{X_{n}}$ can be found by a simple quadrature, once $n-2$ integrals $f_{i}=\alpha_{i}$, (with $i=1, \ldots, n-2$ and constants $\alpha_{1}, \cdots, \alpha_{n-2}$ ) are known. Jacobi remarks that, if a function $M$ satisfies $\nabla \cdot(M \mathbf{X})=0$, by means of the change of variables $V:\left(x_{1}, \cdots, x_{n}\right) \rightarrow$ $\left(\alpha_{1}, \cdots, \alpha_{n-2}, x_{n-1}, x_{n}\right)$ then one can find the $(n-1)$ th integral by a quadrature, and the solution is given by

$$
\int \frac{M X_{n-1} o V^{-1} d x_{n-1}-X_{n} o V^{-1} d x_{n}}{\Delta}=\text { constant }
$$

where $\Delta$ is what is nowadays referred to as the Jacobian determinant of the change of variables. $^{8}$

Jacobi applies his method of the Last Multiplier to Pfaff's problem and to different problems in mechanics. This includes Lagrange's equations, where he takes as auxiliary unknowns the velocity components in order to obtain a first order equation, and applied it also to Hamilton's equations. ${ }^{9}$

Jacobi took a particular interest in Hamilton's theory and contributed to several developments thereof. Hamilton's equations are discussed by Jacobi, in 1837, as a special case of Pfaff's problem. In this context, Jacobi also developed variational methods for different problems in solid mechanics and celestial mechanics. He also developed new methods of resolution of Pfaff's problem, the most important part of which will be published only after his death (in Crelle's Journal 1862, Vol. 60 and in a book based on his lectures in 1866). ${ }^{10}$

The articles written by Clebsch from 1855 to 1860 lay in the perspective opened by the work of Jacobi. Clebsch indeed shows that the equations determining the velocity vector field of a fluid are related to Pfaff's problem. The results of Clebsch about Pfaff's problem were certainly inspired by Jacobi's methods, but can also be considered as a by-product of Clebsch's results on hydrodynamics. ${ }^{11}$

It is namely the methods developed by Jacobi for Pfaff's problem that Clebsch apply in his two articles devoted to hydrodynamics. Clebsch himself will focus on Pfaff's problem in later articles. We keep in mind that the history of Mechanics, during the 19th century, can be seen through the different steps that were required to find the general solution of Pfaff's

\footnotetext{
${ }^{7}$ Kummer 1847.

8 Jacobi, 1844:251.

9 Jacobi 1844:222, 266, 1845:262, 266.

${ }^{10}$ Hamilton 1834, 1835, Jacobi, 1837:128-136, 1862, 1866. For the genesis of Hamilton-Jacobi equations see Nakane-Frazer 2002.

11 Clebsch, 1861, 1862, 1863.
} 
problem, beginning with the work of Jacobi until those of Elie Cartan, including of course the results of Clebsch, Ferdinand Georg Frobenius, Sophus Lie and Jean Gaston Darboux. We do not intend to rediscuss here this process, which is described in detail in the work of Hawkins. We would just like to point out that the articles of Clebsch on hydrodynamics were certainly a source of inspiration for his later works on Pfaff's problem. ${ }^{12}$

We mention, nevertheless, that in his article on Pfaff's problem of 1862, Clebsch develops a method that he names indirect. In his subsequent article on the subject, he gives up this method and finds inspiration from the methods developed by Jacobi in his Nova methodus (Clebsch himself took care of the publication of the latter article by Jacobi in the preceding issue of the Crelle's Journal). In his second paper on Pfaff's problem, Clebsch gives the solution of the problem in dimension $2 n$, stating the integrability conditions. This method by Clebsch, that Elie Cartan will qualify as "very elegant", makes use of the famous brackets introduced by Jacobi. Also on this point, Clebsch can be seen as the direct descendant of Jacobi. A further evidence of the influence of Jacobi on Clebsch's works on hydrodynamics is provided (see Sec. IV.B) by the application, in the 1859 paper, of a canonical transformation in order to set equal to zero a function playing the role of "Hamiltonian". This procedure clearly reminds of what is now known as the Hamilton-Jacobi method. ${ }^{13}$

At the time of Clebsch's two papers on hydrodynamics, work on the fluid dynamics were formulated mainly in Eulerian coordinates. Exceptions were the work of Cauchy of 1815 and of Hankel in 1861, where results on the vorticity are presented in Lagrangian coordinates. ${ }^{14}$

Also, we remark that, after the works of Joseph-Louis Lagrange in 1761-62, the variational approach to hydrodynamics was for a while abandoned. ${ }^{15}$

The purpose of Clebsch in his 1857 paper is to find a variational principle characterising the steady motion of an incompressible fluid. In his second paper of 1859, Clebsch addresses the problem of a variational principle for the unsteady case.

Following an approach typical of pure mathematics and in the tradition of Jacobi, Clebsch, in both papers, considers initially partial differential equations in a generic dimension $(n$ for the 1857 paper and $2 n+1$ in the 1859 paper) and later investigates the applications to fluids in dimension 3. In particular, Clebsch is the first to follow this approach for hydrodynamics.

One has to observe that the practice of treating problems in an arbitrary number of dimensions becomes quite common in the years 1840-50s. For instance, since 1843, Arthur Cayley investigates some problems of analytical geometry in dimension $n$. The first results in the theory of invariants by James Joseph Sylvester and Cayley are disseminated in Germany by means of several articles published by Cayley in Crelle's Journal. The subject of these articles are homogeneous polynomials of several variables. In these articles, Cayley does not restrict the number of variables to three. Moreover, in connection with the theory of invariants, we observe an increased usage of determinants for expressing properties of homogeneous polynomials, and in particular quadratic forms and the associated bilinear forms. Hesse publishes an article about the use of determinants in geometry, where he investigates determinants of order $n$ of symmetric matrices and expresses, in terms of such determinants, what we nowadays refer to as scalar and vector products, namely operations that Clebsch uses in his articles. This is also the time where the Ausdehnungslehre by Hermann Grassmann, which goes unnoticed for a long time, lays the foundations of a geometry in dimension $n$. Finally, the Habilitation thesis by Bernhard Riemann, which will appear only in 1868, defines a local metric on a manifold (although the term Mannigfaltigkeit is due to Carl Friedrich Gauss) of dimension $n .^{16}$

For the variational formulation of the paper of 1857, Clebsch introduces a set of variables $a_{1}, a_{2}, \cdots, a_{n}$, (note that, as detailed below, we use a different notation, with respect to Clebsch, for the functions $a_{i}$ ), alternative to the set of variables given by the $n$ components of the velocity field. Clebsch expresses the components of the velocity field of an incompressible fluid as the co-factors of the element $\partial a_{1} / \partial x_{j}$ of the Jacobian matrix of elements $\partial a_{i} / \partial x_{j}$.

\footnotetext{
12 Hawkins 2005, 2013, Clebsch 1861, 1862, 1863.

13 Hawkins, 2005:395-396, Cartan 1899:240.

14 Cauchy, 1815, Hankel 1861, Frisch-Villone 2014, Frisch-Grimberg-Villone 2017.

15 Lagrange 1760-61:462, where he derives Euler equations for the case of a barotropic fluid.

16 Cayley 1845, 1847, 1851, 1857, Hesse 1855, Grassmann, 1844, Riemann 1854.
} 
This formulation guarantees automatically that the divergence of the velocity field be zero. In particular, in the case $n=3$, the velocity field is expressed as $\mathbf{u}=\nabla a_{2} \times \nabla a_{3}$. In the steady case, considered in the paper of 1857 , the streamlines turn out to be located at the intersection of the hypersurfaces $a_{i}=$ constant, for $i=2, \ldots, n$. These also correspond to integrals of the equations of motion. This kind of representation of the velocity field as the vector product of gradients of scalar functions appears in an article by Euler. In this way Leonhard Euler obtained Bernoulli equation in terms of the streamlines. ${ }^{17}$

In the article of 1859, Clebsch introduces a different expression for the velocity field, which is based on what we now call the celebrated Clebsch variables. In this case Clebsch, in the three-dimensional case, expresses the vorticity field as the product of gradients of such variables. Vorticity lines thus lie at the intersection of hypersurfaces identified by the condition that the "Clebsch variables" be constant. In this representation, the equations of motion for an incompressible fluid can be cast in a form that is reminiscent of the canonical Hamilton's equations of motion. By means of a transformation of variables, Clebsch also shows that the arbitrary function $\Pi$, emerging as a new and undesired degree of arbitrariness in his variational formulation, can be set equal to zero without loss of generality. We remark that Clebsch variables are sometimes referred to as "Monge potentials", following Clifford Truesdell, who credits Gaspard Monge and Johann Friedrich Pfaff for having introduced them, although implicitly, as Truesdell himself points out. Truesdell also establishes the aforementioned connection between Clebsch variables and Pfaff's problem. This connection will be described more in detail later in the paper. ${ }^{18}$

\section{CLEBSCH'S ARTICLE OF 1857 : RESULTS AND METHODS}

Before considering the content of Clebsch's 1857 paper, we find it useful to observe that one of the main difficulties in reading the texts by Clebsch is related to the absence of the concept of vector and of vector analysis. Some of the operations that are nowadays carried out by means of vector analysis, are expressed by Clebsch making use of determinants, and it is not always straightforward to find the corresponding formulation of Clebsch expressions in terms of vector analysis. Therefore, for the sake of a better understanding of the text, we believe it might be useful to follow some parts of Clebsch's article step by step and to reformulate, where possible, his expressions, with the help of vector analysis.

Before proceeding further, it is appropriate to explain the system we adopt in the present paper for referring to equations. Indeed, in the following, we will frequently refer to equations contained in the accompanying articles providing the translation of the two papers by Clebsch. An equation, present in the accompanying articles, which was numbered also in Clebsch original papers, will be referred to as Eq. CT $(n)$, [57.p] if it belongs to the 1857 paper, or as Eq. CT (n), [59.p] if it belongs to the 1859 paper. In these expressions CT is an acronym for "Clebsch Translation". The number $(n)$ indicates the equation number in the original Clebsch paper and the number $p$ corresponds to the additional number adopted in the accompanying paper. So, for instance, Eq. CT (19), [59.35] indicates that we are referring to the equation of the 1859 paper, that Clebsch denoted with the number (19), and that the authors of the accompanying papers denote with [59.35]. Equations that were not numbered by Clebsch will be referred to simply as to Eq. CT [57.p] or CT [59.p], depending on whether they belong to the 1857 or 1859 paper, respectively. Finally, when we refer to equations of the present paper, we use the standard notation Eq. $(n)$, where $n$ is the number of the equation in the present paper.

\section{A. n-dimensional system of equations}

The starting point of Clebsch's analysis consists of a set of partial differential equations in dimension $n$. Clebsch considers the following system (corresponding to CT (1), [57.1])

17 Euler 1757, Truesdell 1954b:XCI-C.

18 Truesdell 1954a:27. 


$$
\nabla V=\frac{\partial \mathbf{u}}{\partial t}+\mathbf{u} \cdot \nabla \mathbf{u}
$$

completed by the following divergence-free condition (corresponding to CT (2), [57.2]), expressing incompressibility in the fluid context:

$$
\nabla \cdot \mathbf{u}=0
$$

We do not know if the initial purpose of Clebsch was to treat Euler's equations in dimension $n$. Clebsch of course is aware of the fact that, for $n=3$, one obtains Euler's equations for an incompressible fluid, and this is in particular the case that he considers in the second part of the article. However, such $n$-dimensional systems are typical of a line of research initiated by Pfaff in 1814 and characterized namely by considering system of equations in a generic dimension $n$. Jacobi also follows this procedure. Indeed, in his articles Jacobi begins with treating systems of differential equations in dimension $n$, before considering the applications to mechanics in dimension $n=3$ (or less). ${ }^{19}$

For the case $n=3$, the vector field $\mathbf{u}$ in Eqs. (2)-(3) corresponds to the fluid velocity field. We remark that the function $V$ includes the pression as well as the potential $U$ associated with external forces which, at that time, were related by $\boldsymbol{F}=\nabla U$, whereas nowadays it is customary to place a minus sign in front of the gradient.

Clebsch introduces an alternative set of variables ${ }^{20}$

$$
a_{1}, a_{2}, \cdots, a_{n}
$$

which allows him to express the components of the velocity vector field as the cofactors of the components of the gradient of the variable $a_{1}$ in the Jacobian matrix associated with transformation

$$
\left(x_{1}, x_{2}, \cdots, x_{n}\right) \mapsto\left(a_{1}, a_{2}, \cdots, a_{n}\right) .
$$

In order to express the velocity vector in modern language, in the form a single determinant, we can consider a basis $\left(e_{i}\right)$ of the Euclidean space of dimension $n$ and write:

$$
\mathbf{u}=\left|\begin{array}{cccc}
e_{1} & e_{2} & \cdots & e_{n} \\
\frac{\partial a_{2}}{\partial x_{1}} & \frac{\partial a_{2}}{\partial x_{2}} & \cdots & \frac{\partial a_{2}}{\partial x_{n}} \\
\cdots & \cdots & \cdots & \cdots \\
\frac{\partial a_{n}}{\partial x_{1}} & \frac{\partial a_{n}}{\partial x_{2}} & \cdots & \frac{\partial a_{n}}{\partial x_{n}}
\end{array}\right|
$$

Making reference to a theorem by Jacobi, Clebsch remarks that this expression for the velocity vector field automatically verifies the equation $\nabla \cdot \mathbf{u}=0$. Indeed, the components of the vector $\mathbf{u}$ so defined, are determinants of $(n-1) \times(n-1)$ matrices whose rows are gradients. $^{21}$

From the variation of the total energy and indicating, as above, with $u_{k}=\Delta_{k}$ the $k$-th component of the velocity vector, Clebsch obtains Eq. CT (10a), [57.11]:

$$
\delta(V-T)=\sum_{k=1}^{k=n}\left(\frac{\partial \Delta_{k}}{\partial t}+M_{k}\right) \delta x_{k}
$$

\footnotetext{
19 Pfaff 1814.

20 Clebsch uses the index notation of Jacobi, $a, a^{\prime}, a^{(2)}, \ldots a^{(n-1)}$. Here we deviate slightly from the original notation by Clebsch and prefer to let the values of the index of the variables go from 1 to $n$.

21 Jacobi 1844:203.
} 
where (see Eq. CT (10), [57.10]):

$$
M_{k}=\sum_{i=1}^{i=n} \Delta_{i}\left(\frac{\partial \Delta_{k}}{\partial x_{i}}-\frac{\partial \Delta_{i}}{\partial x_{k}}\right) .
$$

The vector field $\mathbf{M}$ of components $M_{k}$, is the product of an antisymmetric matrix $N=\left(c_{k j}\right)$ with the velocity vector, so that

$$
\mathbf{M}=N \mathbf{u}
$$

with

$$
c_{k j}=-c_{j k}=\frac{\partial u_{k}}{\partial x_{j}}-\frac{\partial u_{j}}{\partial x_{k}} .
$$

Clebsch realizes also that

$$
\mathbf{u}^{t} \mathrm{M}=\mathbf{u}^{t} N \mathbf{u}=0
$$

From which it follows that, the vector $\mathbf{M}$ being orthogonal to the vector $\mathbf{u}$, it is a linear combination of the vectors $\nabla a_{i}$ for $i=2, \cdots, n$. This yields Eq. CT (11), [57.13] and the system CT (15), [57.18], which is a reformulation of the $n$-dimensional Euler equations in the unsteady case.

We find it also useful to anticipate that, for $n=3$, one has $\mathbf{M}=(\nabla \times \mathbf{u}) \times \mathbf{u}$. The vector field $\mathbf{M}$ then physically corresponds to the cross product of the vorticity with the velocity.

Eqs. CT (11), [57.13] and CT (15), [57.18] by Clebsch, expressed in vector form, read as follows :

$$
\begin{gathered}
\mathbf{M}=\sum_{i=2}^{n} A_{i} \nabla a_{i} . \\
\nabla(V-T)=\sum_{i=2}^{n} A_{i} \nabla a_{i}+\frac{\partial \mathbf{u}}{\partial t} .
\end{gathered}
$$

The latter equation can be written in terms of variations, which corresponds to the form given in Eq. CT (12), [57.14]:

$$
\delta(V-T)=\frac{\partial \mathbf{u}}{\partial t} \cdot \delta \mathbf{x}+\sum_{i=2}^{n} A_{i} \delta a_{i}
$$

In the steady case, it reduces to Eq. CT (13), [57.15]. Namely considering the steady case, Clebsch obtains the identity

$$
\delta(V-T)=\sum_{i=2}^{n} A_{i} \delta a_{i}=\delta \Pi,
$$

where $\Pi=\Pi\left(a_{2}, \cdots, a_{n}\right)$ is an arbitrary function. Upon integration, one may write

$$
V-T=\Pi\left(a_{2}, \ldots, a_{n}\right) .
$$

and for $i=2,3, \cdots n$, one has $A_{i}=\frac{\partial \Pi}{\partial a_{i}}$.

Clebsch has thus shown that, in the time-independent case, the sum of the kinetic $(T)$ and of the potential $(V)$ energy is a function of the quantities $a_{2}, \cdots, a_{n}$ only. In particular, for $n=3$, given that, as above mentioned, streamlines lie at the intersection of surfaces 
$a_{i}=$ constant, it follows that the total energy is constant along the streamlines. Indeed, from the relation

$$
\nabla(V-T)=A_{2} \nabla a_{2}+A_{3} \nabla a_{3}
$$

one obtains $\nabla(V-T) \cdot \nabla a_{2} \times \nabla a_{3}=0$, implying that, with respect to the variables $\left(a_{1}, a_{2}, a_{3}\right)$, the function $V-T$ is a function of $a_{2}$ and $a_{3}$ only. This function is denoted, following Eq. (12), as $\Pi\left(a_{2}, a_{3}\right)$. The streamlines of the velocity field $\mathbf{u}=\nabla a_{2} \times \nabla a_{3}$ lie at the intersections of the surfaces $a_{2}=$ constant and $a_{3}=$ constant, which implies that $\Pi\left(a_{2}, a_{3}\right)$ (and, in turn, the total energy $\left.T-V\right)$ is constant along the streamlines.

When analyzing his Eq. CT (15), [57.18], Clebsch considers determinants that remind of Cramer's formula: the term $S_{h}^{m}$ of the expression CT [57.19] is the $h$ th component of the vector $\mathbf{S}^{m}$.

$$
\mathbf{S}^{m}=\left|\begin{array}{cccc}
e_{1} & e_{2} & \ldots & e_{n} \\
\frac{\partial a_{2}}{\partial x_{1}} & \frac{\partial a_{2}}{\partial x_{2}} & \ldots & \frac{\partial a_{2}}{\partial x_{n}} \\
\frac{\partial a_{m-1}}{\partial x_{1}} & \frac{\partial a_{m-1}}{\partial x_{2}} & \ldots & \frac{\partial a_{m-1}}{\partial x_{n}} \\
\frac{\partial(V-T)}{\partial x_{1}} & \frac{\partial(V-T)}{\partial x_{2}} & \ldots & \frac{\partial(V-T)}{\partial x_{n}} \\
\ldots & \ldots & \ldots & \ldots \\
\frac{\partial a_{n}}{\partial x_{1}} & \frac{\partial a_{n}}{\partial x_{2}} & \ldots & \frac{\partial a_{n}}{\partial x_{n}}
\end{array}\right| .
$$

Because this expression is a multilinear form composed by gradients only, the divergence of the vector field $\mathbf{S}^{m}$ is zero.

When replacing, in the expression for $\mathbf{S}^{m}$, the term $\nabla(V-T)$ by

$$
\nabla(V-T)=\sum_{i=2}^{n} A_{i} \nabla a_{i}+\frac{\partial \mathbf{u}}{\partial t},
$$

Clebsch makes use of the property according to which, when a matrix possesses two identical rows, its determinant vanishes, and obtaines, by linearity of the divergence operator:

$$
0=\nabla \cdot \mathbf{S}^{m}=\nabla \cdot\left(A_{m} \mathbf{u}\right)+\nabla \cdot \mathbf{v}
$$

where the vector $\mathbf{v}$ is equal to

$$
\mathbf{v}=\left|\begin{array}{cccc}
e_{1} & e_{2} & \cdots & e_{n} \\
\frac{\partial a_{2}}{\partial x_{1}} & \frac{\partial a_{2}}{\partial x_{2}} & \cdots & \frac{\partial a_{2}}{\partial x_{n}} \\
\frac{\partial a_{m-1}}{\partial x_{1}} & \frac{\partial a_{m-1}}{\partial x_{2}} & \cdots & \frac{\partial a_{m-1}}{\partial x_{n}} \\
\frac{\partial \Delta_{1}}{\partial x_{1}} & \frac{\partial \Delta_{2}}{\partial x_{2}} & \cdots & \frac{\partial \Delta_{n}}{\partial x_{n}} \\
\cdots & \ldots & \cdots & \ldots \\
\frac{\partial a_{n}}{\partial x_{1}} & \frac{\partial a_{n}}{\partial x_{2}} & \cdots & \frac{\partial a_{n}}{\partial x_{n}}
\end{array}\right| .
$$

The last line in the above expression is namely Eq. CT (17), [57.24] by Clebsch.

Because the divergence of the velocity vector field vanishes, one obtains Eq. CT (18), [57.26] :

$$
0=\mathbf{u} \cdot \nabla A_{m}+\nabla \cdot \mathbf{v}
$$


The term $Q^{m}$ of Clebsch is the last term in this equation. The equations at the end of Sec. 2 of Clebsch's paper are rather simple consequences of Eq. CT (15), [57.18]. In particular, Eq. CT (19), [57.30] extends Eq. CT (14a), [57.17] to the time-dependent case.

Section 3 of Clebsch's article is devoted, on the one hand, to obtaining an expression for the coefficients $A_{m}$ and, on the other hand, to the formulation of a variational principle characterizing the steady state of a fluid. Hereafter we provide a somewhat detailed discussion of some of the steps of Clebsch's calculations, in particular, given that, as already mentioned, his repeated use of determinants, might be unusual for a contemporary reader.

We first remind that Eqs. CT (21), [57.32] correspond to the equations of the system CT (11), [57.13]:

$$
\mathbf{M}=\sum_{i=2}^{n} A_{i} \nabla a_{i} .
$$

Making use of our notation for the indices, Eq. CT (22), [57.32] becomes:

$$
S_{k}=\sum_{i=1}^{i=n} \sum_{h=1}^{h=n} \sum_{m=2}^{m=n} \frac{\partial \Delta_{h}}{\partial x_{i}} \cdot \frac{\partial a_{m}}{\partial x_{k}} \cdot \frac{\partial \Delta_{h}}{\partial \frac{\partial a_{m}}{\partial x_{i}}} .
$$

Clebsch shows that the term $S_{k}$ is equal to $-M_{k}$, where $M_{k}$ is the $k$ th component of the vector $\mathbf{M}$ in Eq. CT (11), [57.13].

In order to understand Clebsch's procedure, one has to note that the term $\partial \Delta_{h} / \partial \frac{\partial a_{m}}{\partial x_{i}}$ is the cofactor of $\frac{\partial a_{m}}{\partial x_{i}}$ in the determinant $\Delta_{h}$. Also, the terms $\frac{\partial a_{m}}{\partial x_{k}} \cdot \partial \Delta_{h} / \partial \frac{\partial a_{m}}{\partial x_{i}}$ can be obtained by replacing the column $\frac{\partial a_{m}}{\partial x_{i}}$ in the determinant $\Delta_{h}$, by the term $\frac{\partial a_{m}}{\partial x_{k}}$, as one calculates the determinant along this column.

Clebsch considers then the result of the sum

$$
\sum_{m=2}^{m=n} \frac{\partial a_{m}}{\partial x_{k}} \cdot \frac{\partial \Delta_{h}}{\partial \frac{\partial a_{m}}{\partial x_{i}}}
$$

as one varies the indices $i$ and $h$ :

- when $i \neq k$ and $h \neq k$, the matrix contains two identical columns, which cancels the determinant;

- when $i=k$, one obtains the determinant $\Delta_{h}$;

- when $h=k$, the determinant $\Delta_{k}$ no longer contains the column $\frac{\partial a_{m}}{\partial x_{k}}$ and adding it amounts to obtaining $-\Delta_{i}$, because, as Clebsch says, removing the $i$ th column in $\Delta_{k}$ amounts to removing the $k$ th column in $\Delta_{i}$, up to the sign.

The above triple sum then yields : $S_{k}=-M_{k}$.

Because the term $S_{k}$ and the $k$ th line of Eq. CT (21), [57.32] are both linear combinations of terms of the form $\frac{\partial a_{m}}{\partial x_{k}}$, it is sufficient to compare the coefficients in order to identify the expression for the $A_{m}$.

One then obtains, using Clebsch's formulation (see Eq. CT (23), [57.37]):

$$
A_{m}=-\sum_{i=1}^{i=n} \sum_{h=1}^{h=n} \frac{\partial \Delta_{k}}{\partial x_{i}} \cdot \frac{\partial \Delta_{h}}{\partial \frac{\partial a_{m}}{\partial x_{i}}} .
$$

Moreover, given that the divergence of the velocity vector field $\mathbf{u}$ is zero, one has

$$
A_{m}=-\sum_{i=1}^{i=n} \frac{\partial}{\partial x_{i}}\left\{\sum_{h=1}^{h=n} \Delta_{h} \frac{\partial \Delta_{h}}{\partial \frac{\partial a_{m}}{\partial x_{i}}}\right\},
$$

which yields Eq. CT (24), [57.41]: 


$$
A_{m}=-\sum_{i=1}^{i=n} \frac{\partial}{\partial x_{i}}\left\{\sum_{h=1}^{h=n} \frac{\partial T}{\partial \frac{\partial a_{m}}{\partial x_{i}}}\right\}
$$

providing the expression for the coefficients $A_{m}$.

After this equation, Clebsch formulates his variational principle : the $n$-dimensional steady Euler equations correspond to the conditions that make the integral

$$
\int(T-\Pi) d x_{1} d x_{2} \ldots d x_{n}
$$

a minimum. We remark that, although the symbol $\Pi$ was previously introduced to indicate a function of the variables $a_{2}, a_{3}, \cdots, a_{n}$, in the latter integral, the function $\Pi$ has to be intended as a function of the variables $x_{1}, x_{2}, \cdots, x_{n}$, according to $\Pi=$ $\Pi\left(a_{2}\left(x_{1}, x_{2}, \cdots, x_{n}\right), a_{3}\left(x_{1}, x_{2}, \cdots, x_{n}\right), \cdots, a_{n}\left(x_{1}, x_{2}, \cdots, x_{n}\right)\right)$.

Minimizing the integral $\int(T-\Pi) d x_{1} d x_{2} \ldots d x_{n}$ amounts to show that the equations following from the condition

$$
\delta \int(T-\Pi) d x_{1} d x_{2} \ldots d x_{n}=0
$$

correspond to

$$
A_{i}=\frac{\partial \Pi}{\partial a_{i}}, \quad \text { for } i=2, \cdots, n .
$$

Clebsch does not prove this principle as, most probably, according to him, its validity follows immediately from Eq. CT (24), [57.41]. A reader familiar with calculus of variations would also find the derivation of this result rather straightforward.

Nevertheless, we provide explicitly some intermediate steps that lead to the result. Actually, one can find an explicit proof (which we follow hereafter) in dimension $n=3$, but easily extendable to an arbitrary dimension $n$. Indeed, here is the argument of Basset. ${ }^{22}$

Given that the kinetic energy density $T$ only depends on the derivatives $\partial a_{i} / \partial x_{j}$, we write

$$
\delta T=\delta T_{2}+\delta T_{3}+\cdots \delta T_{n}
$$

where

$$
\delta T_{i}=\sum_{j=1}^{n} \frac{\partial T}{\partial \frac{\partial a_{i}}{\partial x_{j}}} \delta\left(\frac{\partial a_{i}}{\partial x_{j}}\right), \quad \text { for } i=2, \cdots, n .
$$

We consider then the term

$$
\delta T_{2}=\frac{\partial T}{\partial \frac{\partial a_{2}}{\partial x_{1}}} \delta\left(\frac{\partial a_{2}}{\partial x_{1}}\right)+\frac{\partial T}{\partial \frac{\partial a_{2}}{\partial x_{2}}} \delta\left(\frac{\partial a_{2}}{\partial x_{2}}\right)+\cdots+\frac{\partial T}{\partial \frac{\partial a_{2}}{\partial x_{n}}} \delta\left(\frac{\partial a_{2}}{\partial x_{n}}\right)
$$

We focus on the first term on the right-hand side of this expression. Carrying out an integration by parts, its contribution to $\delta T_{2}$ to the integral $\delta \int(T-\Pi) d x_{1} d x_{2} \ldots d x_{n}$, corresponds to

$$
\begin{aligned}
& \int^{n} \frac{\partial T}{\partial \frac{\partial a_{2}}{\partial x_{1}}} \delta\left(\frac{\partial a_{2}}{\partial x_{1}}\right) d x_{1} d x_{2} \cdots d x_{n} \\
& =\int^{n-1} \frac{\partial T}{\partial \frac{\partial a_{2}}{\partial x_{1}}} \delta a_{2} d x_{2} \cdots d x_{n-1}-\int^{n} \frac{\partial}{\partial x_{1}}\left(\frac{\partial T}{\partial \frac{\partial a_{2}}{\partial x_{1}}}\right) \delta a_{2} d x_{1} d x_{2} \cdots d x_{n}
\end{aligned}
$$

22 Basset 1888:34-38. 
where we added an index $n$ or $n-1$ to the integral symbol, to emphasize that one is integrating over an $n$ or $n-1$ dimensional domain, respectively.

The first term on the right-hand side vanishes, if appropriate boundary conditions are chosen. This is the case, for instance, if one assumes that the variation $\delta a_{2}$ vanishes on the boundary. Although Clebsch does not comment explicitly on the boundary conditions adopted for his variational principles, it appears as if he systematically assumes conditions such that boundary terms vanish when integrating by parts.

Integrating by parts and summing the contributions coming from the other terms in the expression of $\delta T_{2}$, one obtains

$$
\begin{aligned}
& \int \delta T_{2} d x_{1} d x_{2} \cdots d x_{n}=-\int\left(\frac{\partial}{\partial x_{1}} \frac{\partial T}{\partial \frac{\partial a_{2}}{\partial x_{1}}}+\frac{\partial}{\partial x_{2}} \frac{\partial T}{\partial \frac{\partial a_{2}}{\partial x_{2}}}+\ldots+\frac{\partial}{\partial x_{n}} \frac{\partial T}{\partial \frac{\partial a_{2}}{\partial x_{n}}}\right) \delta a_{2} d x_{1} d x_{2} \cdots d x_{n} \\
& =\int A_{2} \delta a_{2} d x_{1} d x_{2} \cdots d x_{n} .
\end{aligned}
$$

Repeating this procedure for the other terms $\delta T_{3}, \cdots, \delta T_{n}$, one can eventually obtain

$$
\begin{aligned}
& \delta \int(T-\Pi) d x_{1} d x_{2} \ldots d x_{n}=\sum_{i=2}^{n} \int\left(\delta T_{i}-\frac{\partial \Pi}{\partial a_{i}} \delta a_{i}\right) d x_{1} d x_{2} \ldots d x_{n} \\
& =\sum_{i=2}^{n} \int\left(A_{i}-\frac{\partial \Pi}{\partial a_{i}}\right) \delta a_{i} d x_{1} d x_{2} \ldots d x_{n}
\end{aligned}
$$

Consequently, imposing $\delta \int(T-\Pi) d x_{1} d x_{2} \ldots d x_{n}=0$ leads to

$$
A_{i}=\frac{\partial \Pi}{\partial a_{i}}, \quad \text { for } i=2, \cdots, n
$$

which completes the proof.

\section{B. 3D equations}

In the case $n=3$, the velocity field in terms of the variables

$$
\left(a_{1}, a_{2}, a_{3}\right)
$$

introduced by Clebsch, is expressed by

$$
\mathbf{u}=\nabla a_{2} \times \nabla a_{3} .
$$

Euler equations of motion for a fluid, on the other hand, read

$$
\nabla V=\partial_{t} \mathbf{u}+\mathbf{u} \cdot \nabla \mathbf{u}
$$

Replacing, in Euler equation, $\mathbf{u} \cdot \nabla \mathbf{u}$ by $(\nabla \times \mathbf{u}) \times \mathbf{u}+\nabla\left(\mathbf{u}^{2} / 2\right)$ one obtains the expression

$$
\nabla(V-T)=\frac{\partial \mathbf{u}}{\partial t}+(\nabla \times \mathbf{u}) \times \mathbf{u}
$$

As above anticipated, the quantity $M_{i}$ introduced by Clebsch, corresponds, in the case $n=3$, to the $i$ th component of the vector field $(\nabla \times \mathbf{u}) \times \mathbf{u}$.

We also have

$$
(\nabla \times \mathbf{u}) \times \mathbf{u}=A_{2} \nabla a_{2}+A_{3} \nabla a_{3},
$$

which makes it possible to calculate directly the following expressions for the coefficients $A_{2}$ and $A_{3}$ : 


$$
A_{2}=(\nabla \times \mathbf{u}) \cdot \nabla a_{3}=\nabla \cdot\left(\mathbf{u} \times \nabla a_{3}\right), \quad A_{3}=-\nabla a_{2} \cdot(\nabla \times \mathbf{u})=\nabla \cdot\left(\mathbf{u} \times-\nabla a_{2}\right) .
$$

Such expressions for the coefficients $A_{2}$ and $A_{3}$ actually appear for the first time already in Euler's works. ${ }^{23}$

Finally, the expressions for the quantities $R_{2}$ and $R_{3}$ by Clebsch, in the case $n=3$, correspond to

$$
R_{2}=\frac{d A_{2}}{d t}=(\nabla \times \mathbf{u}) \cdot \frac{\partial \nabla a_{3}}{\partial t} \quad R_{3}=\frac{d A_{3}}{d t}=-(\nabla \times \mathbf{u}) \cdot \frac{\partial \nabla a_{2}}{\partial t} .
$$

\section{Resolution of the system CT (38), [57.63] by the method of Jacobi's Last Multiplier.}

The equations determining the streamlines of the fluid are reported by Clebsch in his Eq. CT (38), [57.63] and read

$$
\frac{d x_{1}}{d t}=\Delta_{1}, \quad \frac{d x_{2}}{d t}=\Delta_{2}, \quad \frac{d x_{3}}{d t}=\Delta_{3}
$$

This system can also be cast in the form

$$
\frac{d x_{1}}{\Delta_{1}}=\frac{d x_{2}}{\Delta_{2}}=\frac{d x_{3}}{\Delta_{3}}=\frac{d t}{1} .
$$

Clebsch remarks that if, for instance, $a_{3}\left(x_{1}, x_{2}, x_{3}, t\right)=$ constant is an integral of the system, then

$$
\frac{d a_{3}}{d t}=\frac{\partial a_{3}}{\partial t}+\sum_{1}^{3} \Delta_{i} \frac{\partial a_{3}}{\partial x_{i}}=\frac{\partial a_{3}}{\partial t}+\mathbf{u} \cdot \nabla a_{3}=0 .
$$

However, the term $\nabla a_{3} \cdot \mathbf{u}$ vanishes because $a_{3}$ is constant along streamlines. Thus, the partial derivative of $a_{3}$ with respect to time has also to be zero, which implies $R_{2}=0$ or, equivalently, that also $A_{2}=$ constant is an integral of the system.

The system CT (38), [57.63] involves four variables. Thus, as one knows three independent solutions, one knows also the general solution, which is a function $f$ such that the Jacobian of the transformation of $f$ and of the three solutions with respect to the four variables $x_{1}, x_{2}, x_{3}, t$ be zero.

Clebsch then considers two solutions as known and finds the third one by means of the method of Last Multiplier.

More precisely, he supposes to have two solutions $A_{1}=$ constant and $a_{2}=$ constant and wants to determine a third solution $\varphi$, verifying the condition $W=0$, where (see Eq. CT [57.68])

$$
W=\left|\begin{array}{llll}
\frac{\partial \psi}{\partial x_{1}} & \frac{\partial \varphi}{\partial x_{1}} & \frac{\partial a_{2}}{\partial x_{1}} & \frac{\partial A_{1}}{\partial x_{1}} \\
\frac{\partial \psi}{\partial x_{2}} & \frac{\partial \varphi}{\partial x_{2}} & \frac{\partial a_{2}}{\partial x_{2}} & \frac{\partial A_{1}}{\partial x_{2}} \\
\frac{\partial \psi}{\partial x_{3}} & \frac{\partial \varphi}{\partial x_{3}} & \frac{\partial a_{2}}{\partial x_{3}} & \frac{\partial A_{1}}{\partial x_{3}} \\
\frac{\partial \psi}{\partial t} & \frac{\partial \varphi}{\partial t} & \frac{\partial a_{2}}{\partial t} & \frac{\partial A_{1}}{\partial t}
\end{array}\right|
$$

23 Euler 1757:347. 
is the Jacobian determinant of the transformation and $\psi$ is the general solution.

On the other hand, upon carrying out a change of variables from $\left(x_{1}, x_{2}, x_{3}, t\right)$ to $\left(a_{2}, A_{1}, v, t^{\prime}\right)$, where $t^{\prime}=t$ and where $v$ is a fourth independent variable, also the condition

$$
W=\frac{d \psi}{d t}=\frac{\partial \psi}{\partial t}+\frac{\partial \psi}{\partial a_{2}} \frac{d a_{2}}{d t}+\frac{\partial \psi}{\partial A_{1}} \frac{d A_{1}}{d t}+\frac{\partial \psi}{\partial v} \frac{d v}{d t}=0
$$

must hold (note that in this expression we replaced $t^{\prime}$ with $t$ and kept the same symbol $\psi$ for the function $\psi$ written in terms of the the new independent variables).

Because $\frac{d a_{2}}{d t}=0$ and $\frac{d A_{1}}{d t}=0$, this equation reduces to

$$
W=\frac{d \psi}{d t}=\frac{\partial \psi}{\partial t}+\frac{\partial \psi}{\partial v} \frac{d v}{d t}=0
$$

Expressing the Lagrangian derivative $d v / d t$ in terms of the original variables $\left(x_{1}, x_{2}, x_{3}, t\right)$, Clebsch obtains

$$
W=\frac{d \psi}{d t}=\frac{\partial \psi}{\partial t}+\frac{\partial \psi}{\partial v}\left\{\frac{\partial v}{\partial t}+\Delta_{1} \frac{\partial v}{\partial x_{1}}+\Delta_{2} \frac{\partial v}{\partial x_{2}}+\Delta_{3} \frac{\partial v}{\partial x_{3}}\right\}=0 .
$$

The expression for the Jacobian determinant $W$ can also be written in terms of the new variables. Taking into account that $\partial a_{2} / \partial t^{\prime}=\partial A_{1} / \partial t^{\prime}=0$, this leads to

$$
W=D\left|\begin{array}{llll}
\frac{\partial \psi}{\partial a_{2}} & \frac{\partial \varphi}{\partial a_{2}} & 1 & 0 \\
\frac{\partial \psi}{\partial A_{1}} & \frac{\partial \varphi}{\partial A_{1}} & 0 & 1 \\
\frac{\partial \psi}{\partial v} & \frac{\partial \varphi}{\partial v} & 0 & 0 \\
\frac{\partial \psi}{\partial t} & \frac{\partial \varphi}{\partial t} & 0 & 0
\end{array}\right|,
$$

where Clebsch indicates with $D$ the Jacobian determinant of the transformation between the variables $\left(x_{1}, x_{2}, x_{3}, t\right)$ and $\left(a_{2}, A_{1}, v, t^{\prime}\right)$.

In this way Clebsch obtains the identity

$$
\left(\frac{\partial \psi}{\partial t}\right)+\left(\frac{\partial \psi}{\partial v}\right)\left(\frac{\partial v}{\partial t}+\Delta_{1} \frac{\partial v}{\partial x_{1}}+\Delta_{2} \frac{\partial v}{\partial x_{2}}+\Delta_{3} \frac{\partial v}{\partial x_{3}}\right)=D\left|\begin{array}{ll}
\frac{\partial \psi}{\partial v} & \frac{\partial \varphi}{\partial v} \\
\frac{\partial \psi}{\partial t} & \frac{\partial \varphi}{\partial t}
\end{array}\right|
$$

Because this identity must hold for every solution $\psi$, equating the coefficients of $\partial \psi / \partial t$ and $\partial \psi / \partial v$ leads to Eq. CT [57.71]

$$
-1=D \frac{\partial \varphi}{\partial v}, \quad w=D \frac{\partial \varphi}{\partial t}
$$

where

$$
w=\frac{\partial v}{\partial t}+\Delta_{1} \frac{\partial v}{\partial x_{1}}+\Delta_{2} \frac{\partial v}{\partial x_{2}}+\Delta_{3} \frac{\partial v}{\partial x_{3}}
$$

The third integral can then be found, as expected when using the method of Last Multiplier, by quadrature, which yields Clebsch's solution CT (40), [57.72]:

$$
\varphi=\int \frac{w d t-d v}{D}=\text { constant }
$$


2. Formulation in an arbitrary system of coordinates and applications.

Sections 6 and 7 of Clebsch's article of 1857 refer to the case $n=3$ and are devoted to the formulation of his variational principle in an arbitrary system of coordinates and to its application to some special cases, respectively.

At the beginning of Sec. 6 Clebsch introduces a new arbitrary system of time-independent variables denoted as $\left(y_{1}, y_{2}, y_{3}\right)$ and later in the Section derives the expressions required to apply his variational principle in this new system of coordinates. In particular, this amounts to minimize the integral CT [57.83]:

$$
\iiint(T-\Pi) \cdot D d y_{1} d y_{2} d y_{3}
$$

where $T$ and $\Pi$ are expressed in the new coordinates and, according to Eq. CT (44), [57.79],

$$
D=\sqrt{\left|\begin{array}{lll}
u_{11} & u_{12} & u_{13} \\
u_{21} & u_{22} & u_{23} \\
u_{31} & u_{32} & u_{33}
\end{array}\right|}
$$

In the latter expression, the elements $u_{11}, u_{22}, u_{12}, \cdots$ are defined in Eq. CT (52), [57.95], in terms of the (invertible) coordinate transformation $\left(y_{1}, y_{2}, y_{3}\right) \mapsto\left(x_{1}, x_{2}, x_{3}\right)$, by

$$
\sum_{i=1}^{i=3} \frac{\partial x_{i}}{\partial y_{k}} \frac{\partial x_{i}}{\partial y_{h}}=u_{k h}
$$

Clebsch actually introduces the elements $u_{11}, u_{22}, u_{12}, \cdots$ already at the beginning of the Section through the formula CT (43), [57.78]

$$
d s^{2}=u_{11} d y_{1}^{2}+u_{22} d y_{2}^{2}+2 u_{12} d y_{1} d y_{2} \cdots
$$

providing the expression for the square of the line element in the new coordinates.

In our opinion, Sec. 6 contains a detailed derivation, for an arbitrary system of coordinates, of most of the formulas derived by Clebsch in previous Sections, but in general presents no elements of particular historical relevance neither in the methods nor in the results. We nevertheless find it useful to clarify, in the following lines, the derivation of the quantity $P$ in Eq. CT (45), [57.81], by using a more modern approach.

Denoting by $J=\frac{D\left(x_{1}, x_{2}, x_{3}\right)}{D\left(y_{1}, y_{2}, y_{3}\right)}$ the Jacobian matrix associated with the change of coordinates, we remark that the matrix of elements $\left(u_{i j}\right)$ corresponds to the product $J^{T} J$. In fact, upon defining

$$
M=\left(\begin{array}{lll}
u_{11} & u_{12} & u_{13} \\
u_{21} & u_{22} & u_{23} \\
u_{31} & u_{32} & u_{33}
\end{array}\right)
$$

the scalar product of the vector $d \mathbf{x}$ with itself is equal to

$$
d \mathbf{x} \cdot d \mathbf{x}=d \mathbf{y}^{T} M d \mathbf{y}
$$

We also have $d \mathbf{x}=J d \mathbf{y}$, so that

$$
d \mathbf{x} \cdot d \mathbf{x}=(J d \mathbf{y})^{T}(J d \mathbf{y})=d \mathbf{y}^{T} J^{T} J d \mathbf{y},
$$


yielding $M=J^{T} J$.

Considering the function $a=a\left(x_{1}, x_{2}, x_{3}\right)$ and denoting by $\bar{a}\left(y_{1}, y_{2}, y_{3}\right)$ its expression in the coordinates $\left(y_{1}, y_{2}, y_{3}\right)$, we have

$$
\nabla^{x} a=\left(\nabla^{y} a\right)^{T} J^{-1}=\left(J^{-1}\right)^{T} \nabla^{y} \bar{a} .
$$

where we denoted by $\nabla^{x}$ and $\nabla^{y}$ the gradient with respect to the $\left(x_{1}, x_{2}, x_{3}\right)$ and $\left(y_{1}, y_{2}, y_{3}\right)$ coordinates, respectively.

With our notation, the expression for the quantity $P$ introduced by Clebsch in Eq. CT (45), [57.81], reads

$$
\begin{aligned}
P & =\nabla^{x} a_{1} \cdot \nabla^{x} a_{2} \\
& =\left(\left(J^{-1}\right)^{T} \nabla^{y} \bar{a}_{1}\right)^{T}\left(J^{-1}\right)^{T} \nabla^{y} \bar{a}_{2} \\
& =\left(\nabla^{y} \bar{a}_{1}\right)^{T} J^{-1}\left(J^{-1}\right)^{T} \nabla^{y} \bar{a}_{2} .
\end{aligned}
$$

Denoting by $\operatorname{Com} U$ the co-matrix of a generic matrix $U$, we have $J^{-1}=\frac{1}{\operatorname{det} J}(\operatorname{Com} J)^{T}$. It follows then that

$$
J^{-1}\left(J^{-1}\right)^{T}=\frac{1}{D^{2}} \operatorname{Com} M
$$

and finally

$$
P=\frac{1}{D^{2}}\left(\nabla^{y} a_{1}\right)^{T} \operatorname{Com} M \nabla^{y} a_{2} .
$$

The latter expression is consistent with the expression CT (45), [57.81] given by Clebsch, in the form of a determinant.Indeed, when calculating such determinant, the elements of the co-matrix of $M$ appear.

The expression CT (45a), [57.82] for $2 T$ given by Clebsch is based on the work by Hesse cited by Clebsch who expresses, in terms of determinants, the identity ${ }^{24}$

$$
\|\mathbf{u} \times \mathbf{v}\|^{2}=\|\mathbf{u}\|^{2}\|\mathbf{v}\|^{2}-\|\mathbf{u} \cdot \mathbf{v}\|^{2} .
$$

The two applications considered by Clebsch in Sec. 7 concern cases in which the fluid particle motion is independent on one coordinate. In the first case Clebsch considers a velocity field identified by the two functions (again, adopting our notation):

$$
a_{3}=x_{3}, \quad a_{2}=f\left(x_{1}, x_{2}, t\right) .
$$

The components of the velocity field are given by

$$
u_{1}=\Delta_{1}=\frac{\partial a_{2}}{\partial x_{2}}, \quad u_{2}=\Delta_{2}=-\frac{\partial a_{2}}{\partial x_{1}}, \quad u_{3}=\Delta_{3}=0 .
$$

The system is invariant with respect to the $x_{3}$ coordinate. In this case one has, following Eq. CT (57), [57.101], $A_{3}=0$ and

$$
-A_{2}=\frac{\partial^{2} a_{2}}{\partial x_{1}^{2}}+\frac{\partial^{2} a_{2}}{\partial x_{2}^{2}} .
$$

\footnotetext{
${ }^{24}$ Hesse 1855:248.
} 
Therefore, the quantity $A_{2}$ turns out to correspond, in this case, to the fluid vorticity, which, in the two-dimensional limit, has only one finite component, that we denote with $\omega$, given by

$$
\omega=\nabla x_{3} \cdot(\nabla \times \mathbf{u})=-\left(\frac{\partial^{2} a_{2}}{\partial x_{1}^{2}}+\frac{\partial^{2} a_{2}}{\partial x_{2}^{2}}\right) .
$$

The function $a_{2}$, on the other hand, plays the role of what is referred to as a stream function for an incompressible two-dimensional fluid.

The equation describing the dynamics of the fluid, that Clebsch obtains from his Eq. CT (37), [57.61] and that he writes in his Eq. CT (59), [57.103], reads

$$
\frac{\partial a_{2}}{\partial x_{2}} \frac{\partial A_{2}}{\partial x_{1}}-\frac{\partial a_{2}}{\partial x_{1}} \frac{\partial A_{2}}{\partial x_{2}}+\frac{\partial A_{2}}{\partial t}=0
$$

This corresponds to the vorticity equation for a two-dimensional incompressible fluid, where the first two terms are nowadays often written with the help of a canonical Poisson bracket $[$,$] as \left[A_{2}, a_{2}\right]$.

With Eq. CT (60), [57.104] Clebsch remarks that, in the steady case, his variational principle yields

$$
\frac{\partial^{2} a_{2}}{\partial x_{1}^{2}}+\frac{\partial^{2} a_{2}}{\partial x_{2}^{2}}+\Pi^{\prime}\left(a_{2}\right)=0
$$

where $\Pi^{\prime}\left(a_{2}\right)=\frac{\partial \Pi}{\partial a_{2}}$. This translates into the fact that, the vorticity being equal to an arbitrary (invertible) function of the stream function, corresponds to a steady solution for a two-dimensional incompressible fluid. This result was already known to Lagrange and George Stokes . It is however remarkable to note that Clebsch obtains this result from a variational principle. Moreover, making reference to modern Hamiltonian fluid mechanics, the same result can be obtained from the variational principle involved in the Energy-Casimir method $\mathrm{W}$, where one extremizes the functional $F=H+C$, with $H$ being the Hamiltonian functional and $C$ the Casimir invariant. In the case of the two-dimensional Euler equation one has $H=(1 / 2) \int\left|\nabla a_{2}\right|^{2} d x_{1} d x_{2}$ and $C=\int \mathcal{C}(\omega) d x_{1} d x_{2}$, with $\mathcal{C}$ an arbitrary function. Extremizing the functional $F$, while assuming that the first derivative of the function $\mathcal{C}$ be invertible, leads to

$$
\frac{\partial^{2} a_{2}}{\partial x_{1}^{2}}+\frac{\partial^{2} a_{2}}{\partial x_{2}^{2}}+\mathcal{C}^{\prime-1}\left(a_{2}\right)=0
$$

where $\mathcal{C}^{\prime-1}$ indicates the inverse function of the first derivative of $\mathcal{C}$ with respect to its argument. Thus, one sees that the arbitrary function $\Pi$ introduced by Clebsch, in the case when the system is invariant along one direction, is related to the arbitrary function $\mathcal{C}$ associated with the Casimir invariant of the two-dimensional Euler equation. ${ }^{25}$

The second application of Sec. 7 also deals with a system possessing a symmetry along one direction. More precisely Clebsch considers the axisymmetric system in cylindrical coordinates, which allows him also to illustrate the procedure for the change of coordinates that he described in Sec. 6.

\section{CLEBSCH'S ARTICLE OF 1859 : RESULTS AND METHODS}

This article by Clebsch is published after the celebrated article by Hermann von Helmholtz of 1858, which motivated Clebsch to look for a convenient expression for the vorticity.

25 For the two-dimensional case, see Lamb 1895:263. For the Casimir invariant, see Morrison 1998, Holm et al. 1985. 
In particular, Clebsch, whose 1857 article provided a variational principle for the steady case, looks for a new set of variables, in terms of which the condition for the vorticity to be divergence-free is automatically satisfied. This is suitable to provide a variational formulations for the time-dependent equations of motion for an incompressible fluid. This is how Clebsch introduces his new variables, corresponding to those that are nowadays referred to as "Clebsch variables".). In an earlier article, part of Clebsch's 1859 paper was discussed by Frisch-Grimberg-Villone. ${ }^{26}$

Before addressing the general problem in dimension $2 n+1$, in Sec. 1, Clebsch presents, in dimension 3, a change of variable (Eq. CT [59.1]) $(u, v, w) \mapsto(\varphi, m, \psi)$ where $u, v$ and $w$ are the $x, y$ and $z$ components of the velocity vector field, respectively. The transformation to the new variables $(\varphi, m, \psi)$ is such that $u d x+v d y+w d z=d \varphi+m d \psi$. As a consequence, it turns out that the vorticity vector field is given by $\nabla m \times \nabla \psi$.

As Clebsch remarks in Sec. 1, the variational formulation presented in the article of 1859 , permits to cast the Euler equations for an incompressible fluid into a system consisting of two partial differential equations of first order and of a third partial differential equation of second order.

After the introduction Section, Clebsch organizes the presentation of his results in three Sections, which concern: the introduction of the new variables in dimension 3, the derivation of the variational principle in dimension $2 n+1$ and its application to the motion of a fluid (case $n=1$ ), respectively.

\section{A. Clebsch variables in dimension 3}

As mentioned in our previous Section, Clebsch introduces the change of variables $(u, v, w) \mapsto(\varphi, m, \psi)$ such that $u d x+v d y+w d z=d \varphi+m d \psi$. It is however, in a footnote that he explains how the problem of determining the variables $m, \psi$ and $\varphi$, for given $u, v$ and $w$, is related to Pfaff's problem. In Jacobi's 1844 paper, it is explained how to use the last multiplier method for the $n$-dimension problems. On pp. 255-256 this method is reduced to the case of three and four dimensions. Clebsch is not directly quoting from the 1844 paper but from another paper of Jacobi, published in 1846, in which the 1844 paper and various later companion papers were republished. However a minor mistake happened (either to Clebsch or to his printer): the three and four-dimensional cases, which in 1846 had been moved to p. 104, are misquoted as being on p. 144. Later, after the death of Jacobi, the three and four-dimensional cases were republished in 1866 directly by Borchardt notes from Jacobi's oral lectures on Vorlesungen über Dynamik. ${ }^{27}$

If we consider the particular case $(M=1)$ treated by Clebsch, the theorem is the following:

In the case where one can take $M=$ constant, for instance $M=1$, we have the following theorem:

Given a system

$$
\frac{d x}{X}=\frac{d y}{Y}=\frac{d z}{Z}
$$

with $X, Y, Z$ satisfying

$$
\frac{\partial X}{\partial x}+\frac{\partial Y}{\partial y}+\frac{\partial Z}{\partial z}=0
$$

if a solution $\psi=\beta$ is known and if one considers $z$ as a function of $x, y$, and $\beta$ in the expressions for $X, Y$ and $Z$ one obtains the total differential

$$
d m=\frac{1}{\frac{\partial \psi}{\partial z}}(X d y-Y d x)
$$

26 Helmholtz 1858, Frisch-Grimberg-Villone 2017.

27 Jacobi 1844:255-256, 1846:104, Jacobi 1866:77-78. 
One can then determine, by a simple quadrature, the second integral of the system, $m=\alpha$.

We find it useful to provide, hereafter, some details about Jacobi's proof. ${ }^{28}$

For the sake of uniformity in the notation, we adopt, in the following, the symbols used by Clebsch, and not those adopted originally by Jacobi.

First we remark that the case studied by Clebsch corresponds to a case where the multiplier is equal to 1 , because the vector field of components $(X, Y, Z)$, i.e. the vorticity field, is the curl of a vector field and, consequently, its divergence is zero.

Jacobi supposes that $\psi(x, y, z)=\beta$ be an integral of the system

$$
\frac{d x}{X}=\frac{d y}{Y}=\frac{d z}{Z}
$$

and looks for a function $m=m(x, y, z)$ such that $m=\alpha$ be a second integral. He performs a change of coordinates $(x, y, z) \mapsto V(x, y, z)=(x, y, \psi)$ and expresses the gradient of $m$ with respect to the old coordinates, in terms of the new coordinates, which yields:

$$
\left(\begin{array}{l}
\frac{\partial m}{\partial x} \\
\frac{\partial m}{\partial y} \\
\frac{\partial m}{\partial z}
\end{array}\right)=\left(\begin{array}{ccc}
1 & 0 & \frac{\partial \psi}{\partial x} \\
0 & 1 & \frac{\partial \psi}{\partial y} \\
0 & 0 & \frac{\partial \psi}{\partial z}
\end{array}\right)\left(\begin{array}{l}
\frac{\partial(m \circ V)}{\partial x} \\
\frac{\partial(m \circ V)}{\partial y} \\
\frac{\partial(m \circ V)}{\partial \psi}
\end{array}\right)
$$

Jacobi had previously remarked that, if $\psi=\beta$ and $m=\alpha$ are two solutions of the system, then the curl of the vector field associated with the system, i.e. the vorticity field $(X, Y, Z)$ in this case, can be expressed as $\nabla m \times \nabla \psi$. In terms of the new coordinates, this yields

$$
X=\frac{\partial(m \circ V)}{\partial y} \frac{\partial \psi}{\partial z}, \quad Y=-\frac{\partial(m \circ V)}{\partial x} \frac{\partial \psi}{\partial z}, \quad Z=\frac{\partial(m \circ V)}{\partial x} \frac{\partial \psi}{\partial y}-\frac{\partial(m \circ V)}{\partial y} \frac{\partial \psi}{\partial x} .
$$

Therefore one obtains:

$$
\frac{\partial(m \circ V)}{\partial y}=\frac{X}{\frac{\partial \psi}{\partial z}}, \quad \frac{\partial(m \circ V)}{\partial x}=-\frac{Y}{\frac{\partial \psi}{\partial z}} .
$$

Because $d \psi=0$, the differential of $m$, in terms of the new coordinates, reads

$$
d(m \circ V)=\frac{\partial(m \circ V)}{\partial x} d x+\frac{\partial(m \circ V)}{\partial y} d y .
$$

Replacing the partial derivatives of $m \circ V$ by the above expressions, we finally obtain:

$$
d(m \circ V)=\frac{X d y-Y d x}{\frac{\partial \psi}{\partial z}} .
$$

The solution is then given by

$$
m \circ V=\text { constant }=\alpha=\int \frac{X d y-Y d x}{\frac{\partial \psi}{\partial z}} .
$$

28 Jacobi 1866:77. 
Clebsch proceeds in a similar manner in order to determine the functions $m, \psi$ and $\varphi$ such that, for a given velocity vector field of components $u, v$ an $w$, one has $u d x+v d y+w d z=$ $d \varphi+m d \psi$. With regard to $m$ and $\psi$, Clebsch points out that, if one assumes that an integral $\psi$ of the problem

$$
\frac{d x}{X}=\frac{d y}{Y}=\frac{d z}{Z}
$$

with

$$
X=\frac{\partial v}{\partial z}-\frac{\partial w}{\partial y}, \quad Y=\frac{\partial w}{\partial x}-\frac{\partial u}{\partial z}, \quad Z=\frac{\partial u}{\partial y}-\frac{\partial v}{\partial x}
$$

a second integral $m$ can be found by the method of Last Multiplier. We remark that Eqs. (34) and (35) correspond to Eq. CT [59.3]. Because the vorticity field of components $X, Y$ and $Z$ is divergence free, the above described theorem by Jacobi applies. As far as the solution for $\varphi$ is concerned, Clebsch remarks that, because the vector field $m \nabla \psi$ is orthogonal, at each point, to the vorticity vector field, it follows that

$$
\mathbf{u} \cdot(\nabla \times \mathbf{u})=\frac{\partial \varphi}{\partial x} X+\frac{\partial \varphi}{\partial y} Y+\frac{\partial \varphi}{\partial z} Z=u X+v Y+w Z
$$

which corresponds to Eq. CT [59.5].

Recalling that the components of the vorticity fields can be written, in terms of the variables $m$ and $\psi$, as

$$
X=\frac{\partial m}{\partial y} \frac{\partial \psi}{\partial z}-\frac{\partial m}{\partial z} \frac{\partial \psi}{\partial y}, \quad Y=-\frac{\partial m}{\partial x} \frac{\partial \psi}{\partial z}+\frac{\partial m}{\partial z} \frac{\partial \psi}{\partial x}, \quad Z=\frac{\partial m}{\partial x} \frac{\partial \psi}{\partial y}-\frac{\partial m}{\partial y} \frac{\partial \psi}{\partial x}
$$

the above expression can be reformulated as

$$
\left|\begin{array}{lll}
\frac{\partial \varphi}{\partial x} & \frac{\partial \varphi}{\partial y} & \frac{\partial \varphi}{\partial z} \\
\frac{\partial m}{\partial x} & \frac{\partial m}{\partial y} & \frac{\partial m}{\partial z} \\
\frac{\partial \psi}{\partial x} & \frac{\partial \psi}{\partial y} & \frac{\partial \psi}{\partial z}
\end{array}\right|=u X+v Y+w Z .
$$

Clebsch then carries out a change of coordinates $(x, y, z) \mapsto(m, \psi, \theta)$, where $\theta$ is a third, independent coordinate.

We denote by

$$
D=\left|\begin{array}{lll}
\frac{\partial m}{\partial x} & \frac{\partial m}{\partial y} & \frac{\partial m}{\partial z} \\
\frac{\partial \psi}{\partial x} & \frac{\partial \psi}{\partial y} & \frac{\partial \psi}{\partial z} \\
\frac{\partial \theta}{\partial x} & \frac{\partial \theta}{\partial y} & \frac{\partial \theta}{\partial z}
\end{array}\right|
$$

the determinant of the Jacobian matrix associated with this change of variables. Because

$$
\left|\begin{array}{lll}
\frac{\partial \varphi}{\partial m} & \frac{\partial \varphi}{\partial \psi} & \frac{\partial \varphi}{\partial \theta} \\
\frac{\partial m}{\partial m} & \frac{\partial m}{\partial \psi} & \frac{\partial m}{\partial \theta} \\
\frac{\partial \psi}{\partial m} & \frac{\partial \psi}{\partial \psi} & \frac{\partial \psi}{\partial \theta}
\end{array}\right|=\left|\begin{array}{ccc}
\frac{\partial \varphi}{\partial m} & \frac{\partial \varphi}{\partial \psi} & \frac{\partial \varphi}{\partial \theta} \\
1 & 0 & 0 \\
0 & 1 & 0
\end{array}\right|=\frac{\partial \varphi}{\partial \theta},
$$


the above expression, descending from $\mathbf{u} \cdot(\nabla \times \mathbf{u})$, in terms of the new coordinates reads

$$
D \frac{\partial \varphi}{\partial \theta}=u X+v Y+w Z
$$

From this expression one obtains, by integration:

$$
\varphi=\int \frac{u\left(\frac{\partial v}{\partial z}-\frac{\partial w}{\partial y}\right)+v\left(\frac{\partial w}{\partial x}-\frac{\partial u}{\partial z}\right)+w\left(\frac{\partial u}{\partial y}-\frac{\partial v}{\partial x}\right)}{\frac{\partial \theta}{\partial x}\left(\frac{\partial v}{\partial z}-\frac{\partial w}{\partial y}\right)+\frac{\partial \theta}{\partial y}\left(\frac{\partial w}{\partial x}-\frac{\partial u}{\partial z}\right)+\frac{\partial \theta}{\partial z}\left(\frac{\partial u}{\partial y}-\frac{\partial v}{\partial x}\right)} d \theta
$$

which corresponds to the expression CT [59.6] for $\varphi$, given by Clebsch at the end of his footnote.

\section{B. Clebsch variables in dimension $2 n+1$}

In Sec. 2 Clebsch considers what we would call today as the system of Euler equations in dimension $2 n+1$. Clebsch begins with determining, from this system of equations, an expression for $V-T$, (see Eq. CT (6), [59.12]), that can also be obtained by making use of an expression that he derived in his article of 1857. Upon setting

$$
c_{k i}=-c_{i k}=\frac{\partial u_{k}}{\partial x_{i}}-\frac{\partial u_{i}}{\partial x_{k}}
$$

the expression used by Clebsch, taken from his 1857 paper (see Eq. CT (10a), [57.11]) is

$$
\delta(V-T)=\sum_{k=1}^{k=2 n+1}\left(\frac{\partial u_{k}}{\partial t}+\sum_{i=1}^{i=2 n+1} c_{k i} u_{i}\right) \delta x_{k} .
$$

The term involving spatial derivatives can be written in two different ways:

$$
\sum_{i=1}^{i=2 n+1} \sum_{k=1}^{k=2 n+1} c_{k i} u_{i} \delta x_{k}=\sum_{i=1}^{i=2 n+1} \sum_{k=1}^{k=2 n+1} c_{k i} u_{k} \delta x_{i}
$$

This yields Eq. CT (6), [59.12]:

$$
\delta(V-T)=\sum_{k=1}^{2 n+1} \frac{\partial u_{k}}{\partial t} \delta x_{k}+\frac{1}{2} \sum_{i=1}^{i=2 n+1} \sum_{k=1}^{k=2 n+1} c_{k i}\left(u_{i} \delta x_{k}-u_{k} \delta x_{i}\right) .
$$

It is at this point that Clebsch, with his Eq. CT (7), [59.13], introduces $n$ pairs of variables $\left(m_{i}, \varphi_{i}\right)$ in order to represent the velocity field:

$$
\mathbf{u}=\nabla \varphi+\sum_{i=1}^{i=n} m_{i} \nabla \varphi_{i}
$$

This makes it possible for Clebsch to express, in Eq. CT [59.15], the term involving spatial derivatives in $\delta(V-T)$ as a sum of $n$ determinants of the form

$$
\left|\begin{array}{cc}
\mathbf{u} \cdot \nabla m_{r} & \nabla m_{r} \cdot \delta \mathbf{x} \\
\mathbf{u} \cdot \nabla \varphi_{r}, & \nabla \varphi_{r} \cdot \delta \mathbf{x}
\end{array}\right|
$$


Upon replacing the velocity vector field using $\mathbf{u}=\frac{d \mathbf{x}}{d t}$, in the determinants of the form (45), the term involving spatial derivatives becomes, as in Eq. CT [59.16]:

$$
\begin{aligned}
& \frac{1}{2} \sum_{i=1}^{i=2 n+1} \sum_{k=1}^{k=2 n+1} c_{k i}\left(u_{i} \delta x_{k}-u_{k} \delta x_{i}\right) \\
& =\sum_{r=1}^{r=n}\left[\left(\frac{d m_{r}}{d t}-\frac{\partial m_{r}}{\partial t}\right) \delta \varphi_{r}-\left(\frac{d \varphi_{r}}{d t}-\frac{\partial \varphi_{r}}{\partial t}\right) \delta m_{r}\right] .
\end{aligned}
$$

Using again the change of variables (44), the term involving the time derivative in Eq. (43) becomes

$$
\sum_{k=1}^{k=2 n+1} \frac{\partial u_{k}}{\partial t} \delta x_{k}=\delta \frac{\partial \varphi}{\partial t}+\sum_{r=1}^{r=n}\left(m_{r} \delta \frac{\partial \varphi_{r}}{\partial t}+\frac{\partial m_{r}}{\partial t} \delta \varphi_{r}\right),
$$

which corresponds to Eq. CT [59.17].

Finally, by combining the relations (46) and (47) with Eq. (43), Clebsch derives the following relation CT (8), [59.18], which is crucial for establishing his variational principle in the unsteady case:

$$
\delta\left(V-T-\frac{\partial \varphi}{\partial t}-\sum_{r=1}^{r=n} m_{r} \frac{\partial \varphi_{r}}{\partial t}\right)=\sum_{r=1}^{r=n}\left(\frac{d m_{r}}{d t} \delta \varphi_{r}-\frac{d \varphi_{r}}{d t} \delta m_{r}\right)
$$

The quantity on the left-hand side is a total variation. Therefore, the same holds for the right-hand side. More precisely, Clebsch sets the right-hand side equal to $\delta \Pi\left(\varphi_{1}, \cdots, \varphi_{n}, m_{1}, \cdots, m_{n}\right)$, where $\Pi$ is an arbitrary function. From this, Clebsch concludes that the system of Euler's equations in dimension $2 n+1$ can be recast (Theorem 1 ) in the form given by his Eq. CT (9), [59.19], i.e.

$$
\begin{aligned}
& \frac{\partial m_{r}}{\partial t}+u \frac{\partial m_{r}}{\partial x}+u_{1} \frac{\partial m_{r}}{\partial x_{1}}+\cdots=\frac{\partial \Pi}{\partial \varphi_{r}} \\
& \frac{\partial \varphi_{r}}{\partial t}+u \frac{\partial \varphi_{r}}{\partial x}+u_{1} \frac{\partial \varphi_{r}}{\partial x_{1}}+\cdots=-\frac{\partial \Pi}{\partial m_{r}} \\
& \frac{\partial u}{\partial x}+\frac{\partial u_{1}}{\partial x_{1}}+\cdots \frac{\partial u_{2 n}}{\partial x_{2 n}}=0
\end{aligned}
$$

where

$$
u_{k}=\frac{\partial \varphi}{\partial x_{k}}+m_{1} \frac{\partial \varphi_{1}}{\partial x_{k}}+m_{2} \frac{\partial \varphi_{2}}{\partial x_{k}}+\cdots m_{n} \frac{\partial \varphi_{n}}{\partial x_{k}}
$$

and with $r=1, \cdots, n$.

In the new formulation, the system consists of $2 n$ first order partial differential equations evolving $m_{r}$ and $\varphi_{r}$, and of a second order partial differential equation expressing the divergence-free condition. Once the system is solved, the potential energy $V$ can be determined from Eq. CT (10), [59.21], which reads

$$
V=\left(\frac{\partial \varphi}{\partial t}+\sum_{r} m_{r} \frac{\partial \varphi_{r}}{\partial t}\right)+\frac{1}{2} \sum_{k}\left(\frac{\partial \varphi}{\partial x_{k}}+\sum_{r} m_{r} \frac{\partial \varphi_{r}}{\partial x_{k}}\right)^{2}+\Pi
$$

In Eq. CT (11), [59.22] Clebsch points out that the evolution equations for $\varphi_{r}$ and $m_{r}$, presented in Eq. CT (9), [59.19], can be written as

$$
\frac{d \varphi_{r}}{d t}=-\frac{\partial \Pi}{\partial m_{r}}, \quad \frac{d m_{r}}{d t}=\frac{\partial \Pi}{\partial \varphi_{r}}
$$

which is what he calls - at the beginning of Sec. 3 - as the canonical form of the equations. 


\section{The variational principle}

In Theorem 2, Clebsch presents his variational principle, which states that Eqs. CT (9), [59.19] makes the following integral to be a minimum or a maximum:

$$
\int^{2 n+2} V d x_{1} d x_{2} \ldots d x_{2 n+1} d t
$$

Here $V$ is given by the above expression (51), corresponding to Eq. CT (10), [59.21].

We observe that Clebsch does not find it necessary to provide a proof of this result, as he considers it an immediate consequence of previous calculations. Nevertheless, as we did in the case of the variational principle of 1857, we believe it might be useful to give some details about its derivation.

From Eq. CT (8), [59.18], one can derive:

$$
\delta(V-T)=\sum_{r=1}^{r=n}\left[\left(\frac{d m_{r}}{d t}-\frac{\partial m_{r}}{\partial t}\right) \delta \varphi_{r}-\left(\frac{d \varphi_{r}}{d t}-\frac{\partial \varphi_{r}}{\partial t}\right) \delta m_{r}\right]
$$

from which the following identity is readily derived:

$$
\begin{aligned}
& \int^{2 n+2} \delta V d x_{1} d x_{2} \ldots d x_{2 n+1} d t=\int^{2 n+2} \delta T d x_{1} d x_{2} \ldots d x_{2 n+1} d t \\
& +\int^{2 n+2} \sum_{r=1}^{r=n}\left[\left(\frac{d m_{r}}{d t}-\frac{\partial m_{r}}{\partial t}\right) \delta \varphi_{r}-\left(\frac{d \varphi_{r}}{d t}-\frac{\partial \varphi_{r}}{\partial t}\right) \delta m_{r}\right] d x_{1} d x_{2} \ldots d x_{2 n+1} d t .
\end{aligned}
$$

In order to prove the variational principle, it is then required to integrate the variation of the kinetic energy.

One has

$$
\delta T=\sum_{i=1}^{2 n+1} u_{i} \delta u_{i}
$$

If we now consider the first component of the velocity field expressed in terms of Clebsch variables, we obtain

$$
\delta u_{1}=\frac{\partial \delta \varphi}{\partial x_{1}}+\sum_{i=1}^{n} m_{i} \frac{\partial \delta \varphi_{i}}{\partial x_{1}}+\sum_{i=1}^{n} \frac{\partial \varphi_{i}}{\partial x_{1}} \delta m_{i}
$$

Upon multiplying by $u_{1}$, integrating over the whole domain in space and time and carrying out an integration by parts, with respect to the coordinate $x_{1}$, of the first two terms on the right-hand side, we obtain

$$
\begin{aligned}
& \int^{2 n+2} u_{1} \delta u_{1} d x_{1} d x_{2} \cdots d x_{2 n+1} d t=\int^{2 n+1} u_{1}\left(\delta \varphi+\sum_{i=1}^{n} m_{i} \delta \varphi_{i}\right) d x_{2} \cdots d x_{2 n+1} d t \\
& +\int^{2 n+2}\left[u_{1} \sum_{i=1}^{n} \frac{\partial \varphi_{i}}{\partial x_{1}} \delta m_{i}-\frac{\partial\left(m_{i} u_{1}\right)}{\partial x_{1}} \delta \varphi_{i}-\frac{\partial u_{1}}{\partial x_{1}} \delta \varphi_{i}\right] d x_{1} d x_{2} \cdots d x_{2 n+1} d t
\end{aligned}
$$

The first integral on the right-hand side vanishes with appropriate boundary conditions. Thus one has

$$
\begin{aligned}
& \int^{2 n+2} u_{1} \delta u_{1} d x_{1} d x_{2} \cdots d x_{2 n+1} d t \\
& =\int^{2 n+2}\left[u_{1} \sum_{i=1}^{n} \frac{\partial \varphi_{i}}{\partial x_{1}} \delta m_{i}-u_{1} \sum_{i=1}^{n} \frac{\partial m_{i}}{\partial x_{1}} \delta \varphi_{i}-\sum_{i=1}^{n} \frac{\partial u_{1}}{\partial x_{1}}\left(m_{i} \delta \varphi_{i}+\delta \varphi\right)\right] d x_{1} d x_{2} \cdots d x_{2 n+1} d t
\end{aligned}
$$


Repeating the same calculation for all the terms $u_{i} \delta u_{i}$ and summing up the results, one obtains

$$
\begin{aligned}
& \int^{2 n+2} \delta T d x_{1} d x_{2} \ldots d x_{2 n+1} d t= \\
& \int^{2 n+2}\left[\sum_{i=1}^{n}\left(\sum_{i=1}^{n} u_{i} \frac{\partial \varphi_{i}}{\partial x_{i}}\right) \delta m_{i}-\sum_{i=1}^{n}\left(\sum_{i=1}^{n} u_{i} \frac{\partial m_{i}}{\partial x_{i}}\right) \delta \varphi_{i}\right] d x_{1} d x_{2} \ldots d x_{2 n+1} d t \\
& -\int^{2 n+2}\left[\nabla \cdot \mathbf{u} \sum_{i=1}^{n}\left(m_{i} \delta \varphi_{i}+\delta \varphi\right)\right] d x_{1} d x_{2} \ldots d x_{2 n+1} d t
\end{aligned}
$$

The last term vanishes because the divergence of the velocity fields is zero. The two remaining terms let appear the difference between the total Lagrangian derivative and the partial time derivative of the functions $\varphi_{i}$ e $m_{i}$. Thus one has

$$
\begin{aligned}
& \int^{2 n+2} \delta T d x_{1} d x_{2} \ldots d x_{2 n+1} d t= \\
& \int^{2 n+2} \sum_{i=1}^{n}\left[\left(\frac{d \varphi_{i}}{d t}-\frac{\partial \varphi_{i}}{\partial t}\right) \delta m_{i}-\left(\frac{d m_{i}}{d t}-\frac{\partial m_{i}}{\partial t}\right) \delta \varphi_{i}\right] d x_{1} d x_{2} \ldots d x_{2 n+1} d t
\end{aligned}
$$

This expression cancels exactly the right-hand side of the above expression for $\int^{2 n+2} \delta V d x_{1} d x_{2} \ldots d x_{2 n+1} d t$, showing that the latter functional is extremized when Eqs. CT (9), [59.19] are satisfied. This variational principle is cited, among others, in works by Hicks, Basset, Truesdell and Toupin. ${ }^{29}$

We already remarked, similarly to what done in the case of the variational principle of 1857, that Clebsch most probably finds such algebraic steps rather evident and consequently does not illustrate them. On the other hand, we found that Basset offers a rather detailed derivation of Clebsch's variational principle. In particular, Basset uses also the fact that the arbitrary function $\Pi$ can be taken to be the zero function, provided one carries out the appropriate canonical transformation.

This property is demonstrated by Clebsch in Sec. 3, where he introduces a new set of $n$ variables $a_{1}, \cdots, a_{n}$ and a function $(W)=(W)\left(\varphi_{1}, \cdots, \varphi_{n}, a_{1}, \cdots, a_{n}, t\right)$ such that

$$
\Pi=\left(\frac{\partial W}{\partial t}\right)
$$

and that

$$
m_{r}=\left(\frac{\partial W}{\partial \varphi_{r}}\right), \quad-\alpha_{r}=\left(\frac{\partial W}{\partial a_{r}}\right)
$$

are integrals of

$$
\frac{d m_{r}}{d t}=-\frac{\partial \Pi}{\partial \varphi_{r}}, \quad \frac{d \varphi_{r}}{d t}=\frac{\partial \Pi}{\partial m_{r}} .
$$

The last two expressions correspond to Eqs. CT (13), [59.25] and CT (12), [59.24], respectively. The symbols $\alpha_{1}, \cdots \alpha_{n}$ indicate new constants. Here we also followed the notation adopted by Clebsch, who encloses between parentheses ( ) the function $W$, and its derivatives, when these are considered as functions of the variables $\varphi_{1}, \cdots, \varphi_{n}, a_{1}, \cdots, a_{n}, t$, in order to distinguish them from $W$, and its derivatives, considered as functions of the variables $x_{1}, \cdots, x_{2 n}, t$. In the latter case, the standard notation $W, \partial W / \partial x_{r}, \cdots$ is used, to indicate $W$ and its derivatives.

${ }^{29}$ Hicks 1882:59, Basset 1888:30, Truesdell-Toupin 1860:427. 
In terms of the new variables, the potential $V$ reads, according to Eq. CT (15), [59.30]:

$$
V=\frac{\partial(\varphi+W)}{\partial t}+\alpha_{1} \frac{\partial a_{1}}{\partial t}+\alpha_{2} \frac{\partial a_{2}}{\partial t}+\ldots+\frac{1}{2} \sum_{r=1}^{r=n}\left(\frac{\partial(\varphi+W)}{\partial x_{r}}+\alpha_{1} \frac{\partial a_{1}}{\partial x_{r}}+\alpha_{2} \frac{\partial a_{2}}{\partial x_{r}}+\ldots\right)^{2} .
$$

Comparing this expression with the previous expression, given in Eq. (10), one sees that, by carrying out the replacements

$$
\left(\varphi, \varphi_{i}, m_{i}\right) \longmapsto\left(\varphi+W, a_{i}, \alpha_{i}\right)
$$

the expression for $V$ no longer depends on the arbitrary function $\Pi$, which can then be set equal to zero, without loss of generality. Such transformation that eliminates the arbitrary function in equations in canonical form appears also in other contexts, and can be related to what is nowadays referred to as the Hamilton-Jacobi method. ${ }^{30}$

Clebsch concludes then with Eq. CT (16), [59.31], that the variables $a_{i}$ and $\alpha_{i}$ satisfy the equations

$$
\frac{d a_{i}}{d t}=0, \quad \frac{d \alpha_{i}}{d t}=0
$$

In his Theorem 3 Clebsch provides, by renaming the variables according to the above replacements, a new reformulation of the Euler's equations in dimension $2 n+1$, which is equivalent to the previous one, but where $\Pi=0$. It follows that $2 n$ integrals of motion are given by

$$
m_{r}=\text { constant }, \quad \varphi_{r}=\text { constant }
$$

and the remaining integral can be found by the method of Last Multiplier. In his Theorem 4 he states the variational principle associated with this new formulation.

Section 5 is finally devoted to an application of the general results to the three-dimensional case $(n=1)$, where, as seen above, $u d x+v d y+w d z=d \varphi+m d \psi$. In particular, Clebsch concludes that the system

$$
\frac{d x}{d t}=u, \quad \frac{d y}{d t}=v, \quad \frac{d z}{d t}=w
$$

admits

$$
m=\text { constant }, \quad \psi=\text { constant }
$$

as integrals, as stated in Eq. CT (23), [59.41]. We recall that the intersections of the surfaces $m=$ constant and $\psi=$ constant identify vortex lines. The third integral, as above stated, can be found by the method of Last Multiplier.

The functions $m, \psi$ and $\varphi$ satisfy the following system CT (24), [59.42]

$$
\begin{aligned}
& \frac{\partial m}{\partial t}+\left(\frac{\partial m}{\partial x} \frac{\partial \varphi}{\partial x}+\frac{\partial m}{\partial y} \frac{\partial \varphi}{\partial y}+\frac{\partial m}{\partial z} \frac{\partial \varphi}{\partial z}\right)+m\left(\frac{\partial m}{\partial x} \frac{\partial \psi}{\partial x}+\frac{\partial m}{\partial y} \frac{\partial \psi}{\partial y}+\frac{\partial m}{\partial z} \frac{\partial \psi}{\partial z}\right)=0, \\
& \frac{\partial \psi}{\partial t}+\left(\frac{\partial \psi}{\partial x} \frac{\partial \varphi}{\partial x}+\frac{\partial \psi}{\partial y} \frac{\partial \varphi}{\partial y}+\frac{\partial \psi}{\partial z} \frac{\partial \varphi}{\partial z}\right)+m\left(\frac{\partial \psi}{\partial x} \frac{\partial \psi}{\partial x}+\frac{\partial \psi}{\partial y} \frac{\partial \psi}{\partial y}+\frac{\partial \psi}{\partial z} \frac{\partial \psi}{\partial z}\right)=0, \\
& \frac{\partial}{\partial x}\left(\frac{\partial \varphi}{\partial x}+m \frac{\partial \psi}{\partial x}\right)+\frac{\partial}{\partial y}\left(\frac{\partial \varphi}{\partial y}+m \frac{\partial \psi}{\partial y}\right)+\frac{\partial}{\partial z}\left(\frac{\partial \varphi}{\partial z}+m \frac{\partial \psi}{\partial z}\right)=0 .
\end{aligned}
$$

30 Jacobi 1890:137-138, 1890:393, Lanczos, 1970:238, Landau and Lifshitz, 1976:148. 
This system can be rewritten in the following more compact form:

$$
\begin{aligned}
\frac{\partial m}{\partial t} & =-\mathbf{u} \cdot \nabla m, \\
\frac{\partial \psi}{\partial t} & =-\mathbf{u} \cdot \nabla \psi, \\
\nabla^{2} \varphi & =-\nabla \cdot(m \nabla \psi) .
\end{aligned}
$$

As is well known, one of the most remarkable features of Clebsch variables is that they make Euler's equations for an incompressible fluid amenable to a canonical Hamiltonian formulation. ${ }^{31}$

Indeed, the first two equations of the above system can be written as

$$
\frac{\partial m}{\partial t}=\frac{\delta H}{\delta \psi}, \quad \frac{\partial \psi}{\partial t}=-\frac{\delta H}{\delta m},
$$

where

$$
H=\frac{1}{2} \int|\nabla \varphi+m \nabla \psi|^{2} d x d y d z
$$

is the Hamiltonian functional. In the expression for $H, \varphi$ has to be written in terms of $m$ and $\psi$ using the divergence-free condition, corresponding to the third equation of the system. The Clebsch variables $m$ and $\psi$ are then canonically conjugate field variables.

\section{CONCLUSIONS}

The point in common between the articles of Clebsch of 1857 and 1859 is the search for a variational formulation of Euler equations for an incompressible fluid. In the first article Clebsch expresses the velocity field as the vector product of two gradients, whereas in the second paper it is the vorticity field which admits such a representation. The latter formulation makes it possible for Clebsch to provide a variational formulation for unsteady cases. The "Clebsch variables" introduced in this case, will also make it possible to cast Euler's equations in canonical Hamiltonian form. One of the most striking aspects of both articles is the intention of Clebsch of placing Euler's equations for a three-dimensional fluid, in a more general setting, namely a space of dimension $n$ in the 1857 paper and a space of dimension $2 n+1$ in the 1859 paper. This occurred at a time where some concepts of differential geometry (differential operators, tangent space) were still at an infant stage. Thus, with Clebsch, hydrodynamics becomes a particular case of a general setting as it was the case for solid mechanics in the work of Jacobi.

It is also worth commenting about the influence that the two articles by Clebsch (in particular, the 1859 one) had later on the research in the 20th and 21st centuries.

Concerning the 1857 paper, the main connection with more recent results is probably related to the variational principle leading to equilibrium equations for an incompressible fluid. As already mentioned in Sec. III.B.2, this is related to the variational derivation of equilibrium equations for noncanonical Hamiltonian systems. ${ }^{32}$

Much more considerable, in our opinion, are the repercussions, on contemporary research, of the 1859 article. One of the most relevant references in this respect, as already mentioned in Sec. I, is the one by Seliger and Whitham, which elucidates the role of Clebsch variables in formulating variational principles for continuum mechanics and electromagnetism. In particular, Seliger and Whitham find a connection between Clebsch's variational principle and a constrained Hamilton's variational principle. This can be illustrated more clearly

31 See, for instance, Kuznetsov and Mikhailov 1980, Morrison 1998 and Gallavotti 2010.

32 See, e.g. Morrison 1998 and Holm et al. 1985. 
considering the case of a barotropic compressible fluid (the incompressible case treated by Clebsch follows in a straightforward way). Indeed, one can consider the functional

$$
S=\int\left[\rho \frac{u^{2}}{2}-\rho \epsilon(\rho)+\phi\left(\frac{\partial \rho}{\partial t}+\nabla \cdot(\rho \mathbf{u})\right)+\psi\left(\frac{\partial(\rho m)}{\partial t}+\nabla \cdot(\rho m \mathbf{u})\right)\right] d x_{1} d x_{2} d x_{3} d t
$$

where $\rho=\rho\left(x_{1}, x_{2}, x_{3}, t\right)$ is the mass density of the fluid and $\epsilon$ is the internal energy per unit mass, a given function of $\rho$. Note that the functional $S$ has the form of a constrained action functional, such as those used in Hamilton's principle. Indeed, the sum of the first two terms is of the form $T-\mathcal{V}$, with the first term indicating the kinetic energy density $T$ and the second term representing the potential energy density $\mathcal{V}$. The remaining terms yield the constraints. In particular, setting to zero $\delta S / \delta \phi$, i.e. the functional derivative of $S$ with respect to $\phi$, yields as constraint the continuity equation

$$
\frac{\partial \rho}{\partial t}+\nabla \cdot(\rho \mathbf{u})=0
$$

whereas $\delta S / \delta \psi=0$, combined with Eq. (67), yields

$$
\frac{\partial m}{\partial t}+\mathbf{u} \cdot \nabla m=0
$$

corresponding to Eq. (63), i.e., the evolution equations for the Clebsch variable $m$. The remaining relations following from Hamilton's principle, and corresponding to

$$
\frac{\delta S}{\delta \mathbf{u}}=0, \quad \frac{\delta S}{\delta m}=0, \quad \frac{\delta S}{\delta \rho}=0,
$$

yield

$$
\begin{aligned}
& \mathbf{u}=\nabla \phi+m \nabla \psi \\
& \frac{\partial \psi}{\partial t}+\mathbf{u} \cdot \nabla \psi=0 \\
& \frac{u^{2}}{2}-\epsilon-\rho \frac{d \epsilon}{d \rho}-\left(\frac{\partial \phi}{\partial t}+\mathbf{u} \cdot \nabla \phi\right)-m\left(\frac{\partial \psi}{\partial t}+\mathbf{u} \cdot \nabla \psi\right)=0
\end{aligned}
$$

respectively.

Equation (70) is namely the representation of the velocity field in terms of Clebsch variables, which is then seen to follow from the constrained Hamilton's principle (note that, without the constraints, one would simply get the trivial case $\mathbf{u}=0$ ). Equation $(71)$ corresponds to the evolution equation for the second Clebsch variable $\psi$, whereas Eq. (72) provides a generalized Bernoulli principle. We note that Seliger and Whitham associate the origin of the constraints in the action functional (66) with the so called Lin's constraints , expressing the conservation of the Lagrangian labels along particle trajectories. Moreover, the Lagrangian density appearing under the integral in Eq. (66) is shown by Seliger and Whitham to correspond, up to the sign, to the pressure for an isentropic fluid (Seliger and Whitham actually consider the more general case of an adiabatic fluid). By means of this identification one can retrieve, as above anticipated, the incompressible case originally treated by Clebsch, given that the function $V$ in CT (10), [59.21] is namely (in the absence of external forces) the expression for the pressure in the incompressible case. ${ }^{33}$

In terms of repercussions on the research of the last decades, one of the most remarkable properties of Clebsch variables is that they make it possible to cast the Euler equation in a canonical Hamiltonian form. Indeed, if one considers the functional

$$
H=\frac{1}{2} \int u^{2} d x_{1} d x_{2} d x_{3}=\frac{1}{2} \int(\nabla \phi+m \nabla \psi)^{2} d x_{1} d x_{2} d x_{3}
$$

33 Lin 1963. 
corresponding to the total kinetic energy, the evolution equations for the Clebsch variables $m$ and $\psi$ can be written in the form

$$
\begin{aligned}
& \frac{\partial m}{\partial t}=\frac{\delta H}{\delta \psi}=-\mathbf{u} \cdot \nabla m \\
& \frac{\partial \psi}{\partial t}=-\frac{\delta H}{\delta m}=-\mathbf{u} \cdot \nabla \psi
\end{aligned}
$$

where the incompressibility condition $\nabla \cdot(\nabla \phi+m \nabla \psi)=0$ was also used. ${ }^{34}$

In the early 1980s, canonical Hamiltonian formulations of fluid models in terms of Clebsch variables (as opposed to the noncanonical Hamiltonian formulation provided for hydrodynamics and magnetohydrodynamics by Morrison and Greene) are formulated in the work by Morrison and in that by Kuznetsov and Mikhailov. The canonical formulation in terms of Clebsch variables plays also a fundamental role for the modern study of nonlinear wave interactions in hydrodynamics and plasma dynamics, as reviewed in the work by Falkovich and L'vov, in the one by Zakharov, Musher and Rubenchik, and also in the paper by Zakharov and Kuznetsov. ${ }^{35}$

Indeed, canonical variables allow for the application of canonical transformations, by means of which one can systematically obtain Hamiltonian equations describing the evolution of waves subject to weak nonlinear interactions (this approach actually does not use directly the variables $m$ and $\psi$ but the Fourier coefficients of the complex conjugate fields $a$ and $a^{*}$, where $\left.a=(m+i \psi) \sqrt{2}\right)$. A more recent investigation in this direction concerns the formulation, in terms of Clebsch variables, of Hall magnetohydrodynamics, for the purpose of studying weak turbulence theory in space plasmas. ${ }^{36}$

The connection between the canonical equations (74)-(75) and the variational principle stated in Theorem 2 by Clebsch is further elucidated by Rund, who also proves a theorem generalizing such connection. In the case $n=3$ such theorem states that the equations

$$
\begin{aligned}
& \rho\left(\frac{d m}{d t}-\frac{\partial \Pi}{\partial \psi}\right)=0 \\
& \rho\left(\frac{d \psi}{d t}+\frac{\partial \Pi}{\partial m}\right)=0 \\
& \frac{\partial \rho}{\partial t}+\sum_{j=1}^{3} \frac{\partial}{\partial x^{j}}\left(\rho \frac{\partial H}{\partial u_{j}}\right)=0
\end{aligned}
$$

are equivalent to the system of equations extremizing the integral $\int \mathcal{L}_{0} d x^{1} d x^{2} d x^{3} d t$, with Lagrangian density

$$
\mathcal{L}_{0}=\rho\left(\frac{\partial \phi}{\partial t}+m \frac{\partial \psi}{\partial t}+H\left(x^{1}, x^{2}, x^{3}, u_{1}, u_{2}, u_{3}, t\right)+\Pi(m, \psi, t)\right) .
$$

In Eq. (76)-(78) an auxiliary field $\rho\left(x^{1}, x^{2}, x^{3}, t\right)$ was introduced. It clearly corresponds to the mass density in the above example by Seliger and Whitham, but in general it corresponds to a scalar field satisfying a continuity equation of the form (78). In Eq. (79) $H$ is the Hamiltonian of a canonical Hamiltonian system with generalized coordinates $x^{1}, x^{2}, x^{3}$ and momenta $u_{1}, u_{2}, u_{3}$. Considering $x^{1}, x^{2}, x^{3}$ as local coordinates of a three-dimensional manifold, the variables $u_{1}, u_{2}, u_{3}$ can be seen as the components of a type $(0,1)$ tensor field related to the Clebsch variables $\phi, m, \psi$ by the Clebsch transformation $\mathbf{u}=\nabla \phi+m \nabla \psi$. Note that, following Rund, we adopted here the lower index notation for the component of the velocity field, as opposed to the upper index, in order to indicate the geometric nature of the vector field $\mathbf{u}$ which is that of a $(0,1)$ tensor field. Note that, when the auxiliary field

\footnotetext{
34 Kuznetsov and Mikhailov 1980.

35 Falkovich and L'vov 1995, Zakharov, Musher and Rubenchik 1985, Zakharov and Kuznetsov 1997.

36 Sahraoui, Belmont and Rezeau 2003.
} 
$\rho$ is taken as a constant and $\sum_{j=1}^{3} \partial H / \partial u_{j}=0$ (which is the case when the fluid is incompressible and $\left.H=(1 / 2) \sum_{j=1}^{3} u_{j}^{2}\right)$, the Lagrangian density $\mathcal{L}_{0}$ corresponds to the function $V$ appearing in Eq. CT (10), [59.21] and present in the Theorem 2 by Clebsch. The general proof by Rund permits then to extend the result by Clebsch to more general Hamiltonian systems, by associating them with variational principles. Rund finds applications of this result also to the dynamics of charged particles in the presence of electromagnetic fields. The same Author also develops, with the help of Clebsch variables, a variational formulation of electromagnetism in the presence of magnetic charges. ${ }^{37}$

Canonical Clebsch variables have also inspired transformations that allow, as explained by Morrison, to construct canonical variables from a noncanonical Hamiltonian system based on a Lie-Poisson structure. Following such reference we consider a finite-dimensional LiePoisson system of dimension $N .^{38}$

If the Lie bracket of the system is of the form

$$
[f, g]=w_{k} c_{i j}^{k} \frac{\partial f}{\partial w_{i}} \frac{\partial g}{\partial w_{j}}, \quad i, j, k=1, \cdots N,
$$

with $c_{i j}^{k}$ indicating the structure constants of the underlying Lie algebra and $w_{1}, \cdots, w_{N}$ the noncanonical variables, one can construct a canonical description in terms of conjugate variables $q^{1}, \cdots, q^{N}, p_{1}, \cdots, p_{N}$ related to the noncanonical variables by

$$
w_{i}=c_{i j}^{k} p_{k} q^{j} .
$$

The Lie-Poisson bracket associated with the variables $w_{i}$ and with the Lie algebra with structure constants $c_{i j}^{k}$ becomes a canonical bracket when expressed in terms of the variables $p_{k}$ and $q_{j}$.

This result can be extended to the infinite-dimensional case. One can consider, for instance, Euler equation for an incompressible two-dimensonal fluid expressed in terms of the vorticity $\omega(x, y, t)=\hat{z} \cdot \nabla \times \mathbf{u}(x, y, t)$. Such equation reads

$$
\frac{\partial \omega}{\partial t}+[\varphi, \omega]=0
$$

where $\varphi$ is the stream function related to the vorticity by $\omega=\left(\partial_{x x}+\partial_{y y}\right) \varphi$. In this case the Lie bracket is given by

$$
[f, g]=\frac{\partial f}{\partial x} \frac{\partial g}{\partial y}-\frac{\partial f}{\partial y} \frac{\partial g}{\partial x}
$$

The corresponding canonical variables are two fields $P$ and $Q$, related to the noncanonical variable $\omega$ by

$$
\omega=[Q, P]=\hat{z} \cdot \nabla Q \times \nabla P .
$$

In the case of a three-dimensional fluid, one can take as the noncanonical variables the three components $M_{i}=\rho u_{i}$, for $i=1,2,3$, of the fluid momentum M. In this case, the canonical variables $P_{i}$ and $Q^{j}$ are related to the noncanonical variables by

$$
M_{i}=\sum_{j=1}^{3} P_{j} \frac{\partial Q^{j}}{\partial x^{i}}+\frac{\partial\left(P_{i} Q^{j}\right)}{\partial x^{j}} .
$$

The first term on the right-hand side of Eq. (85) is analogous to the term $m \nabla \psi$ involving namely the canonical variables in Clebsch representation of the velocity field. The transformation (85), on the other hand, is clearly more general than that introduced by Clebsch, for it involves three pairs of canonically conjugate variables and an additional term on the

\footnotetext{
37 Rund 1977a, 1977b.

38 Morrison 1998.
} 
right-hand side. However, analogously to the original Clebsch transformation, also the mapping (85) is not injective. Such extensions of Clebsch transformation inspired further works. For instance, by means of an analogous procedure, Morrison and Hazeltine provide the canonical Hamiltonian formulation for reduced magnetohydrodynamics, whereas Morrison, Caldas and Tasso extend such formulation to account for gyroviscosity in plasmas. Still in the context of the relation of Clebsch variables with Lie algebras. Marsden and Weinstein elucidate the role of Clebsch variables in the geometric formulation of incompressible Euler equation with applications also to two-dimensional fluids and $N$-point vortex models. In the context of geometric mechanics, Marsden, Ratiu and Weinstein also develop the introduction of Clebsch variables in the reduction of Hamiltonian systems on the dual of a Lie algebra of a semidirect product, with applications to compressible fluids and elasticity. Cendra and Marsden propose variational principles based on Clebsch variables and reduction theory, whereas Holm and Kupershmidt derive noncanonical Poisson brackets for magnetohydrodynamics, multifluid plasmas and elasticity models starting from canonical brackets in Clebsch variables. On the other hand, Zakharov derives conserved functionals for the equations of motion of a two-dimensional incompressible fluid expressed in terms of Clebsch variables. ${ }^{39}$

An issue that we already briefly mentioned, concerns the injectivity of the map $\left(u_{1}, u_{2}, u_{3}\right) \rightarrow(\phi, m, \psi)$ expressing Clebsch transformation. The representation of a divergence-free vector field in terms of Clebsch variables is indeed not unique. This issue is discussed by Yoshida, who also discusses the problem of completeness of the Clebsch variables, i.e. of whether a generic vector field $\mathbf{u}(x, y, z)$ with three components, can be represented as $\mathbf{u}=\nabla \varphi+m \nabla \psi$, with three scalars $\varphi, m$ and $\psi$. Clebsch representation turns out not to be complete and a more general representation $\mathbf{u}=\nabla \varphi+\sum_{j=1}^{\nu} m_{j} \nabla \psi_{j}$, with $\nu$ pairs of scalars $\left(m_{j}, \psi_{j}\right)$, is required, where $\nu=n-1$ and $n$ indicates the space dimension. ${ }^{40}$

To conclude, we mention that Clebsch variables or extensions thereof, found applications also in further contexts. For instance, Grad and Rubin make use of a modified Clebsch representation of a magnetic field for investigating nonlinear partial differential equations describing magnetohydrodynamic equilibria relevant for plasma confinement in fusion devices. Extensions of the Clebsch representation of vector fields are also introduced by Mendes et al. in order to treat relativistic hydrodynamics, whereas further examples of such extensions are applied to fluid models in field theories, in the work by Jackiw, Nair and So-Young $\mathrm{Pi}$ and also in the work by Jackiw and Polychronakos. Another application of an extension of Clebsch variables concerns the problem of quantizing Hamiltonian fluid mechanics, as in the work by Ghosh. Incompleteness of the Clebsch parametrization in relation with variational principles involving Maxwell and Chern-Simons actions is discussed in the article by Deser, Jackiw and Polychronakos. Multiple Clebsch variables for the representation of incompressible fluid velocity fields are also considered in an article by Balkovsky. ${ }^{41}$

For further applications of Clebsch variables in the context of fluid dynamics we refer the reader to the recent review by Scholle, Marner and Gaskell. ${ }^{42}$

As mentioned in Sec. I, it is an unrealistic task to provide an exhaustive list of all the papers that make use of Clebsch variables. We hope, however, that the above references will be sufficient to give the reader at least a flavor of the importance that the variables introduce by Clebsch in his 1859 paper had and still have on different branches of theoretical physics and mathematics.

\section{ACKNOWLEDGEMENTS}

GG and ET are grateful to Uriel Frisch, without whom the realization of this article would

\footnotetext{
39 Morrison 1982, Morrison and Greene 1980, Kuznetsov and Mikhailov 1980, Morrison and Hazeltine 1984, Morrison, Caldas and Tasso, 1984, Marsden and Weinstein 1983, 1984, Cendra and Marsden 1987, Holm and Kupershmidt 1983, Zakharov, 1989.

40 Yoshida 2009.

41 Grad and Rubin 1958, Mendes et al. 2005, Jackiw, Nair and So-Young Pi 2000, Jackiw and Polychronakos 2000, Ghosh 2002, Deser, Jackiw and Polychronakos 2001, Balkovsky 1994.

42 Scholle, Marner and Gaskell 2020.
} 
not have been possible. GG and ET are also thankful to the two Reviewers, who provided useful and constructive comments which helped improving the paper. GG wishes to thank the Observatoire de la Côte d'Azur and the Laboratoire J.-L. Lagrange for their hospitality and financial support.

\section{References}

Balkovsky, E. 1994. Some notes on the Clebsch representation for incompressible fluids. Physics Letters A, 186 issue 1-2: 135-136.

Basset, A.B. 1888. A Treatise on Hydrodynamics, London. Reprinted by Dover Publications 1961.

Bateman, H. 1929. Notes on a Differential Equation Which Occurs in the Two-Dimensional Motion of a Compressible Fluid and the Associated Variational Problems. Proc. R. Soc. Lond., 125 issue 799: 598-618.

Beltrami, E. 1871. Sui principi fondamentali della idrodinamica. Mem. Acad.Sci. Bologna, 1, 1871: 431-476; 2, 1872: 381-437; 3 1873: 349-407; 5 1874: 443-484, Richerche sulla cinematica dei fluidi Opere Matematiche Tome 2 Milano 1904: 202-379.

Bretherton, F. 1970. A note on Hamilton's principle for perfect fluids. J. Fluid Mechanics, 44: 1, $19-31$.

Burau, W. 1970-1980. Clebsch, Rudolf Friedrich Alfred. In Dictionary of scientific biography, 3: 313-315, Charles Scribner Sons, New York.

Calkin, M.G. 1963. An Action Principle for Magnetohydrodynamics. Canadian Journal of Physics, 41: 1, 2241-2251.

Cartan, E. 1899. Sur certaines expressions différentielles et le problème de Pfaff. Annales scientifiques de L'E.N.S., 3ème Ser., 16: 1, 239-332.

Cauchy, A-L. 1815/1827. Théorie de la propagation des ondes à la surface d'un fluide pesant d'une profondeur indéfinie - Prix d'analyse mathématique remporté par M. Augustin-Louis Cauchy, ingénieur des Ponts et Chaussées. (Concours de 1815). Mémoires présentés par divers savans à l'Académie royale des sciences de l'Institut de France et imprimés par son ordre. Sciences mathématiques et physiques. Tome I, imprimé par autorisation du Roi à l'Imprimerie royale: $5-318$.

Cayley, A. 1845. Chapters in the analytical geometry of $\mathrm{n}$ dimensions. Cambridge Mathematical Journal IV: 119-127.

Cayley, A. 1847. Recherches sur l'élimination, et sur la théorie des courbes. J. Reine Angew. Math. 34: $34-49$.

Cayley, A. 1851. Note sur la thorie des Hyperdterminants. J. Reine Angew. Math. 42: 368-371.

Cayley, A. 1857. Mémoire sur la forme canonique des fonctions binaires. J. Reine Angew. Math. 54: 48-58, 292.

Cendra, H., Marsden, J.E. 1987. Lin constraints, Clebsch potentials and variational principles. Physica D 27, 63-89.

Clebsch, A. 1854. De motu ellipsoidis in fluido incompressibili viribus quibuslibet impulsi. Dissertatio Inaugurali Physico-Matematica, P.P.O F. E. Neumann, Regiomonti PR, impressit Ernestus Julius Daldowski.

Clebsch, A. 1856. Über die Bewegung eines Ellipsoids in einer tropfbaren Flüssigkeit. J. Reine Angew. Math. 52: 103-132.

Clebsch, A. 1857a. Über die Bewegung eines Ellipsoids in einer tropfbaren Flüssigkeit, Note zu der Abhandlung im Band LII dieses Journals. J. Reine Angew. Math. 53: 293-297.

Clebsch, A. 1857b. Über eine allgemeine Transformation der hydrodynamischen Gleichungen. $J$. Reine Angew. Math. 54: 293-312.

Clebsch, A. 1858a. Über die Reduction der zweiten Variation auf ihre einfachste Form. J. Reine Angew. Math. 55: 254-273.

Clebsch, A. 1858b. Über diejenigen Probleme der Variationsrechnung, welche nur eine unabhaängige Variable enthalten. J. Reine Angew. Math. 55: 335-355.

Clebsch, A. 1858c. Über die Criterien des Maximums und des Minimums in der Variationsrechnung. Monatsberichte der Königlichen Preuss. Akademie der Wissenschaften zu Berlin Aus dem Jahre 1857, Königlichen Akademie der Wissenschaften, 1858: 618-621.

Clebsch, A. 1859a. Über die Integration der hydrodynamischen Gleichungen. J. Reine Angew. Math. 56: 1-10.

Clebsch, A. 1859b. Üeber die zweite Variation vielfacher Integrale. J. Reine Angew. Math. 56: $122-48$.

Clebsch, A. 1861. Über Jacobis Methode, die partiellen Differentialgleichungen erster Ordnung zu integriren und ihre Ausdehnung auf das Pfaffsche Problem, Auszug aus einem Schreiben an den Herausgeber. J. Reine Angew. Math. 59: 190-192. 
Clebsch, A. 1862. Über das Pfaffsche Problem. J. Reine Angew. Math. 60: 193-251.

Clebsch, A. 1863. Über das Pfaffsche Problem. Zweite Abhandlung. J. Reine Angew. Math. 61: 146-179.

Deser, S., Jackiw, R. and Polychronakos, A.P., 2001. Clebsch (string) decomposition in d=3 field theory. Phys. Lett. A, 279 151-153.

Dictionary of scientific biography 1970-1980, 16 vol, ed. Charles Coulston Gillipsie, Charles Scribner Sons, New York.

Duhem P. 1901. Sur les équations de l'hydrodynamique. Commentaire à un mémoire de Clebsch. Annales de la faculté des sciences de Toulouse 2e série, tome 3, no 2 (1901): 253-279.

Euler, 1757. Continuation des recherches sur la théorie du mouvement des fluides. Mémoires de l'académie des sciences de Berlin, Volume 11: 316-361. In Euler, Opera omnia, ser. 2, 12 (Lausanne), pp. $92-132$.

Falkovich, G. and L'vov V.S., 1995. Isotropic and Anisotropic turbulence in Clebsch variables. Chaos, Solitons and Fractals, 5 1855-1869.

Frisch U. and B. Villone 2014. Cauchy's almost forgotten Lagrangian formulation of the Euler equation for 3D incompressible flow. The European Physical Journal H. 39: 325-351.

Frisch, U., Grimberg, G., Villone, B. 2017 A contemporary look at Hermann Hankel's 1861 pioneering work on Lagrangian Fluid dynamics. The European Physical Journal H. 42: 537-546.

Gallavotti, G. 2010. Foundations of Fluid Dynamics, Springer-Verlag, Berlin, Heidelberg.

Ghosh, S. 2002. 'Gauging' the fluid. J. Phys. A: Math. Gen., 3510747.

Grad, H. and Rubin, H., 1958. Hydromagnetic equilibria and force-free fields, Proceedings of the Second United Nation Conference on Peaceful Uses of Atomic Energy, Geneva, 1958, Vol. 31, p. 190.

Grassmann, H.G. 1844. Die Lineale Ausdehnungslehre, ein neuer Zweig der Mathematik, Wiegand, Leipzig.

Hamilton, W.R. 1834. On a General Method in Dynamics. Phil.Transactions of the Royal Society Part 2: 247-308.

Hamilton, W.R. 1835. Second Essay on a General Method in Dynamics. Phil. Trans. of the R. Soc. Part 1: 95-144.

Hankel, H. 1861. Zur allgemeinen Theorie der Bewegung der Flüssigkeiten. Eine von der philosophischen Facultät der Georgia Augusta am 4. Juni 1861 gekrönte Preisschrift, Göttingen.

Hawkins, G. 2005. Frobenius, Cartan, and the Problem of Pfaff. Arch. Hist. Exact Sci. , 59 381-436, Springer, New York.

Hawkins, G. 2013. The Problem of Pfaff. In The Mathematics of Frobenius in Context, 155-204, Springer, New York.

Helmholtz, H. 1858. Über Integrale der hydrodynamischen Gleichungen, welche den Wirbelbewegungen entsprechen. J. Reine Angew. Math. 55: 25-55.

Herivel, J. W. 1954. A General Variational Principle for Dissipative Systems: II. Proceedings of the Royal Irish Academy. Section A: Mathematical and PhysicalSciences, Vol. 56 (1953/1954), 56 (1953/1954): 67-75.

Herivel, J.W. 1955. The derivation of the equations of motion of an ideal fluid by Hamilton's principle. Mathematical Proceedings of the Cambridge Philosophical Society, 51, 2 issue 799: 344-349.

Hesse, O. 1855. Über Determinanten und ihre Anwendung in der Geometrie, insbesondere auf Curven vierter Ordnung. J. Reine Angew. Math. 49: 243-264.

Hicks, W. M. 1882. Report on Recent Progress in Hydrodynamics, Part 1. Report of the Fifty-First Meeting of the Britisch association for the advancement of Science Held at York in August and September 1881 London, 1882: 57-88.

Holm D. D., Kupershmidt B. A., 1983. Poisson brackets and Clebsch representations for magnetohydrodynamics, multifluid plasmas, and elasticity. Physica D, 6 : 347-363.

Holm D. D., Marsden J. E., Ratiu T., Weinstein A. 1985. Nonlinear stability of fluid and plasma equilibria. Phys. Reports, 123 1-116.

Jackiw, R., Nair, V.P., So-Young Pi, 2000. Chern-Simons reduction and non-Abelian fluid mechanics. Phys. Rev. D, 62085018.

Jackiw, R. and Polychronakos, A.P., 2000. Supersymmetric fluid mechanics. Phys. Rev. D, 62 085019.

Jacobi, C.G.J. 1837. Über die Reduction der Integration der partiellen Differentialgleichungen erster Ordnung zwischen irgend einer Zahl Variabeln auf die Integration eines einzigen Systems gewöhnlicher Differentialgleichungen. J. Reine Angew. Math. 17: 1-189.

Jacobi, C.G.J. 1844. Theoria novi multiplicatoris systemati aequationum differentialium vulgarium applicandi. J. Reine Angew. Math. 27: 199-268.

Jacobi, C.G.J. 1845. Theoria novi multiplicatoris systemati aequationum differentialium vulgarium applicandi. J. Reine Angew. Math. 29: 213-279.

Jacobi, C.G.J. 1846. Mathematische Werke, Band I. Berlin. 
Jacobi, C.G.J. 1851. Mathematische Werke, Band II. Berlin.

Jacobi, C.G.J. 1862 Nova methodus, aequationes differentiales partiales primi ordinis inter numerum variabilium quemcunque propositas integrandi. J. Reine Angew. Math. 60: 1-181.

Jacobi, C.G.J. 1866. Vorlesungen über Dynamik. Herausgegeben von A. Clebsch, Reimer, Berlin.

Jacobi, C.G.J. 1890. Gesammelte Werke Band 5 Berlin.

Kummer, E.E. 1847. Zur Theorie der complexen Zahlen. J. Reine Angew. Math. 35: 319-326.

Kuznetsov E.A., Mikhailov, A.V. 1980. On the topological meaning of canonical Clebsch variables. Physics Letters A 77, 1: 5, 3986-3989.

Lagrange, J.L. 1760-1761. Application de la méthode exposé dans le Mémoire précédent à la solution de différents problémes de dynamique, Miscellanea Taurinensia, Oeuvres. 1: 365-468.

Lamb H. 1895. Hydrodynamics, first edition, Cambridge.

Lanczos, C. 1970. The Variational Principles of Mechanics, Dover Publications, New York.

Landau, L.D. and E.M. Lifshitz 1976 Mechanics, Course of Theoretical Physics, 1, Third Ed. Elsevier.

Lin, C.C. 1963. Liquid Helium, Proc. Int. School of Physics, Course XXI, Academic Press, New York.

Marsden, J., and Weinstein A., 1983. Coadjoint orbits, vortices, and Clebsch variables for incompressible fluids. Physica D: Nonlinear Phenomena, 7, 1-3, 305-323.

Marsden, J., Ratiu T., and Weinstein A., 1984. Semidirect Products and reduction in Mechanics. Transactions of the American Mathematical Society, 281, 1, 147-177.

Mendes, A.C.R., Neves, C., Oliveira, W. and Takakura, F.I., 2005. Hidden symmetries in (relativistic) hydrodynamics. J. Phys. A: Math. Gen., 38, 1, 8747-8762.

Merzbach, U. 2018 Dirichlet: A Mathematical Biography, Birkhauser.

Morrison, P.J. and J. M. Greene 1980. Noncanonical Hamiltonian density formulation of hydrodynamics and ideal magnetohydrodynamics. Phys. Rev. Lett. 45: 790-794.

Morrison, P. J. 1982. Poisson Brackets for Fluids ans Plasmas in Mathematical Methods, Hydrodynamics and Integrability in Related Dynamical Systems, AIP Conference Proceedings No. 88 edited by M. Tabor and Y.Treve (AIP, New York):13-46.

Morrison, P.J. and R.J. Hazeltine. 1984. Hamiltonian Formulation of reduced magnetohydrodynamics. Phys. Fluids 27: 886-897.

Morrison P.J., Caldas I.L. and H. Tasso. 1984. Hamiltonian formulation of two-dimensional gyroviscous MHD. Zeitschrift für Naturforschung 39a: 1023-1027.

Morrison, P.J. 1998. Hamiltonian description of the ideal fluid. Rev. Mod. Phys. 70: 467-521.

Nakane, M. and C.G. Fraser. 2002. The Early History of Hamilton-Jacobi Dynamics 18341837. Centaurus 44: 161-227.

Pfaff, J.F. 1814. Methodus generalis, aequationes differentiarum partialium nec non aequationes differentiales vulgates, ultrasque primi ordinis, inter quotcunque variables, complete integrandi. Abhandlungen der Königlichen Akademie der Wissenschaften in Berlin, 76-136.

Riemann, B. 1854. Über die Hypothesen, welche der Geometrie zugrunde liegen., Abh. Kgl. Ges. Wiss., Gttingen 1868.

Rund, H. 1977a. Clebsch potentials in the theory of electromagnetic fields admitting electric and magnetic charge distributions. J. Math. Phys. 18, 84.

Rund, H. 1977b. Clebsch potentials and variational principles in the theory of dynamical systems. Archive for Rational Mechanics and Analysis 65, 305-334.

Sahraoui, F., Belmont, G. and Rezeau, L. 2003. Hamiltonian canonical formulation of Hallmagnetohydrodynamics: Toward an application to weak turbulence theory. Phys. Plasmas 10, 1325.

Scholle, M., Marner, F. and P.H Gaskell. 2020. Potential fields in fluid mechanics: A review of two classical approaches and related recent advances. Water 12, 1241.

Seliger, R.L. and G.B. Whitham. 1968. Variational Principles in Continuum Mechanics. Proc. R. Soc. Lond. A 305, 1-25,305: 1-25

Truesdell, C. 1954a. The Kinematics of Vorticity, Indiana University Science Series no. 19. Indiana University Press (Bloomington).

Truesdell, C. 1954b. 'Rational fluid mechanics, 1657-1765.' In Euler, Opera omnia, ser. 2, 12 (Lausanne), IX-CXXV.

Truesdell, C., Toupin, R.A., 1960 Classical Fields Theory, in Encyclopedia of Physics, ed. S. Flugge, Vol. III/1, Principles of Classical Mechanics and Field Theory, Springer Verlag, pp. 226-793.

Various Authors 1873a. Zum Andenken an Rudolf Friedrich Alfred Clebsch. Mathematische Annalen, 6: 197-202.

Various Authors 1873b. Versuch einer Darlegung und Würdigung seiner wissenschaftlichen Leistungen von einigen seiner Freunde: "R.F. Alfred Clebsch's Mathematische Arbeiten", Mathematische Annalen 7: 1-50.

Yoshida, Z. 2009. Clebsch parametrization: Basic properties and remarks on its applications. J. Math. Phys., 50: 113101. 
Zakharov, V.E., 1989. The algebra of integrals of motion of two-dimensional hydrodynamics in Clebsch variables. Functional Analysis and Its Applications, 23, 3: 189-196.

Zakharov, V.E., Musher, S.L. and A.M. Rubenchik. 1985. Hamiltonian approach to the description of non-linear plasma phenomena. Physics Reports, 129, 5: 285-366.

Zakharov, V. E., and E.A. Kuznetsov. 1997. Hamiltonian formalism for nonlinear waves. Physics Uspekhi, 40, 11: 1087. 